Pacific Journal of Mathematics

ENUMERATING IMMERSIONS AND EMBEDDINGS OF 


\section{ENUMERATING IMMERSIONS AND EMBEDDINGS OF PROJECTIVE SPACES}

\section{L. LARMORE AND R. D. RIGdON}

o. Introduction and statement of results. Let $\left[M \subset R^{n}\right]$ and $\left[M \subseteq R^{n}\right]$ denote, respectively, the set of isotopy classes of embeddings and regular homotopy classes of immersions of the $m$-dimensional manifold $M$ in $n$-dimensional Euclidean space $R^{n}$. Certain maps arise naturally in the study of $\left[M \subset R^{n}\right]$ and $\left[M \subseteq R^{n}\right]$. These maps are

$$
\begin{aligned}
& \phi_{n}:\left[M \subset R^{n}\right] \rightarrow\left[M \subseteq R^{n}\right] \\
& \mathscr{I}_{n}:\left[M \subseteq R^{n}\right] \rightarrow\left[M \subseteq R^{n+1}\right] \\
& \mathscr{E}_{n}:\left[M \subset R^{n}\right] \rightarrow\left[M \subset R^{n+1}\right]
\end{aligned}
$$

which are obtained, respectively, by regarding an embedding as an immersion, an immersion in $R^{n}$ as an immersion in $R^{n+1}$, and an embedding in $R^{n}$ as an embedding in $R^{n+1}$.

Let $P^{m}$ be real projective $m$-space. The main purpose of this paper is to determine the following diagram for $m \geqq 8$ :

$$
\begin{gathered}
{\left[P^{m} \subset R^{2 m}\right] \stackrel{\phi_{2 m}}{\longrightarrow}\left[P^{m} \subseteq R^{2 m}\right]} \\
\uparrow \mathscr{E}_{2 m-1} \\
{\left[P^{m} \subset R^{2 m-1}\right] \stackrel{\phi_{2 m-1}}{\longrightarrow}\left[P^{m} \subseteq \mathscr{I}_{2 m-1}\right.} \\
\left.\uparrow R^{2 m-1}\right] \\
\uparrow \mathscr{E}_{2 m-2} \\
{\left[P^{m} \subset R^{2 m-2}\right] \stackrel{\phi_{2 m-2}}{\longrightarrow}\left[P^{m} \subseteq R^{2 m-2}\right.}
\end{gathered}
$$

The paper is divided into two parts. In $\S \S 1-3$, we review twisted cohomotopy theory and describe the results of Haefliger, Hirsch, Becker, McClendon, and others which reduce the study of $\left[M \subset R^{n}\right]$ and $\left[M \subseteq R^{n}\right]$ to the study of cohomotopy groups. In $\S \S 4-6$, we calculate diagram (0.1).

For more extensive calculations of immersion groups of projective spaces, see Robinson [18]. Many of the results of this paper have been obtained independently by David Bausum in [1] and [2].

The main results are as follows. For $2 n>3(m+1),\left[M \subset R^{n}\right]$ naturally has the structure of an Abelian affine group, while if $2 n>$ $3 m+1,\left[M \subseteq R^{n}\right]$ is an Abelian affine group [3]. The functions $\phi_{n}, \mathscr{E}_{n}$, and $\mathscr{I}_{n}$ are each affine morphisms whenever both domain and target are 
affine groups, and an Abelian affine group becomes an Abelian group by choice of zero. Thus, if an embedding $f: P^{m} \rightarrow R^{2 m-2}$ is chosen, (0.1) becomes a diagram of Abelian groups and homomorphisms.

THEOREM 0.1. Let $m \geqq 8$, such that $P^{m} \subset R^{2 m-2}$. Fix such an embedding. Then

Case $I . \quad m \equiv 0 \bmod 4$. Then diagram $(0.1)$ becomes



Case II. $m \equiv 1 \bmod 4$. Then diagram (0.1) becomes



where

$$
\begin{array}{ll}
\mathscr{C}_{2 m-2}(1,0,0)=\mathscr{C}_{2 m-2}(0,1,0)=0, & \mathscr{E}_{2 m-2}(0,0,1)=1 \\
\mathscr{I}_{2 m-1}(1,0,0)=\mathscr{I}_{2 m-1}(0,1,0)=0, & \mathscr{I}_{2 m-1}(0,0,1)=1 \\
\mathscr{I}_{2 m-2}(1,0)=(1,0,0), & \mathscr{I}_{2 m-2}(0,1)=(0,1,0) \\
\phi_{2 m-1}(1)=(0,1,0) & \\
\phi_{2 m-2}(1,0,0)=\phi_{2 m-2}(0,1,0)=(0,0), & \phi_{2 m-2}(0,0,1)=(0,1)
\end{array}
$$

Case III. $m \equiv 2 \bmod 4$. Diagram (0.1) becomes

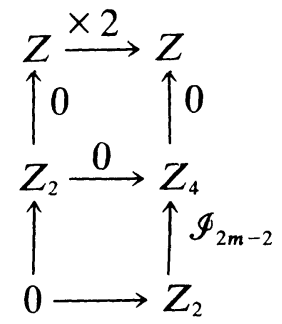

where $\mathscr{I}_{2 m-2}$ is mono. 
Case $I V . \quad m \equiv 3 \bmod 4 . \quad$ Diagram (0.1) becomes

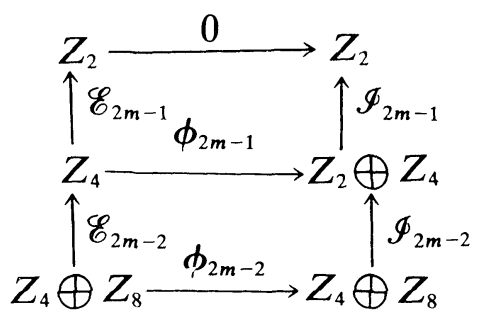

where

$$
\begin{aligned}
& \mathscr{E}_{2 m-1}(1)=1 \\
& \mathscr{E}_{2 m-2}(1,0)=1 \quad \text { and } \quad \mathscr{E}_{2 m-2}(0,1)=0 \\
& \mathscr{I}_{2 m-1}(1,0)=0 \quad \text { and } \quad \mathscr{I}_{2 m-1}(0,1)=1 \\
& \mathscr{I}_{2 m-2}(1,0)=(1,0) \quad \text { and } \mathscr{I}_{2 m-2}(0,1)=(0,1) \\
& \phi_{2 m-1}(1)=(1,0) \\
& \phi_{2 m-2}(1,0)=(1,0) \quad \text { and } \phi_{2 m-2}(0,1)=(0,0) \text { or }(2,0)
\end{aligned}
$$

It is well-known that $P^{m}$ embeds in $R^{2 m-1}$ unless $m$ is a power of 2 and in $R^{2 m}$ in all cases. By Mahowald [13] and Handel [8], $P^{m}$ embeds in $R^{2 m-2}$ if and only if $m=2^{r}+s$ with $2 \leqq s<2^{r}$. Hence the results of Theorem 0.1 apply to the whole of diagram $(0.1)$ for $m>9$ and $m=2^{r}+s, 2 \leqq s<2^{r}$; to the top square of $(0.1)$ for $m>5$ and $m$ not a power of 2 , and to the top line for any $m>3$.

To resolve the uncertainty in $\phi_{2 m-2}$ when $m \equiv 3 \bmod 4$, the following problem must be solved: is $\operatorname{ker}\left(\mathscr{E}_{2 m-2}\right)=\operatorname{ker}\left(\phi_{2 m-2}\right)$ ? That is, is it true that two embeddings $P^{m} \rightarrow R^{2 m-2}$ are isotopic as embeddings in $R^{2 m-1}$ if and only if they are regularly homotopic as immersions in $R^{2 m-2}$ ? The authors have been unable to solve this problem.

1. Twisted cohomotopy. We generally use the notation of [10]; the results of that paper carry over to weak fibrations [5]. The theorems and constructions in this section are essentially the same as those done by Becker [4] for vector bundles.

Let $p: Y \rightarrow X$ be a weak $k$-sphere fibration, for some $k \geqq-1$ (where $S^{-1}$ is the empty set). If $A \subset X$, we define $\pi_{p}^{i}(X, A)$, the $i$ th cohomotopy group of $(X, A)$ twisted by $p$, for any integer $i$, to be the direct limit

$$
\pi_{p}^{i}(X, A)=\operatorname{Lim}_{n \rightarrow \infty}\left[X, A ; \Omega_{X}^{n} \Sigma_{X}^{n+i-k-1} S_{X} Y\right]
$$

where $S_{X}$ and $\Sigma_{X}$ are the fiberwise two-point (unreduced) and fiberwise 
one-point (reduced) suspensions, respectively, and $\Omega_{X}$ is the fiberwise loop.

Twisted cohomotopy satisfies all the usual axioms for a twisted cohomology theory, since $\pi_{p}^{i}(X, A)=H^{i-k}(X, A ; \mathscr{E}(p))$, where $\mathscr{E}(p)$ is the $X$-spectrum associated with $p[10]$.

If $p$ is the sphere bundle associated with a real vector bundle $\xi$, we write $\pi_{\xi}^{i}(X, A)$ for $\pi_{p}^{i}(X, A)$. Note that in that case, in the terminology of McClendon [14], $\pi_{\xi}^{i}(X, A)$ is a cohomology group in the category of spaces over $B O$. In fact, in the notation of Becker [4], $\pi_{\xi}^{i}(X, A)=$ $H^{i}(X, A, f ; \mathscr{S})$, where $\mathscr{S}$ is the sphere spectrum, and $f: X \rightarrow B O$ classifies $\xi$.

Clearly, an equivalence of weak sphere fibrations induces an isomorphism of cohomotopy groups twisted thereby. More strongly:

THEOREM 1.1. For $p: Y \rightarrow X$ and $p^{\prime}: Y^{\prime} \rightarrow X$ are stably equivalent weak sphere fibrations, then $\pi_{p}^{i}(X, A)$ and $\pi_{p^{\prime}}^{i}(X, A)$ are isomorphic for each integer $i$.

Proof. It is sufficient to consider the case $Y^{\prime}=S_{X} Y$. The collapsing map $S_{X} Y^{\prime}=S_{X} S_{X} Y \rightarrow \Sigma_{X} S_{X} Y$, an equivalence of weak $(k+2)$-sphere fibrations, induces the desired isomorphism (where $k=\operatorname{dim} p$ ), and we are done.

Whitney sum and cup product. Let $p: Y \rightarrow X$ and $p^{\prime}: Y^{\prime} \rightarrow X$ be weak $k$-sphere and $k^{\prime}$-sphere fibrations respectively. We define the Whitney sum $p \oplus p^{\prime}=p *_{X} p^{\prime}: Y *_{X} Y^{\prime} \rightarrow X$, a weak $\left(k+k^{\prime}+1\right)$-sphere fibration over $X$ (where $*_{X}$ is the fiberwise join). The Whitney sum notation is justified by the fact that if $p$ and $p^{\prime}$ are the sphere bundles associated to vector bundles $\xi$ and $\xi^{\prime}, p \oplus p^{\prime}$ is the sphere bundle associated to the usual Whitney sum $\xi \oplus \xi^{\prime}$.

For any integers $i$ and $i^{\prime}$, and for any $A, A^{\prime} \subset X$, we define a cup product:

$$
\pi_{p}^{i}(X, A) \times \pi_{p^{\prime}}^{i^{\prime}}\left(X, A^{\prime}\right) \rightarrow \pi_{p+p^{\prime}}^{i+i^{\prime}}\left(X, A \cup A^{\prime}\right)
$$

as follows. If $a \in \pi_{p}^{i}(X, A)$ and $a^{\prime} \in \pi_{p^{\prime}}^{i^{\prime}}\left(X, A^{\prime}\right)$ are represented, respectively, by $\alpha: X \rightarrow \Omega_{X}^{n} \sum_{X}^{n+i-k-1} S_{X} Y$ and $\alpha^{\prime}: X \rightarrow \Omega_{X}^{n^{\prime}} \sum_{X}^{n^{\prime}+i^{\prime}-k^{\prime}-1} S_{X} Y^{\prime}$, let $(-1)^{n} a \cup a^{\prime}$ be represented by the composition

$$
\begin{gathered}
X \stackrel{\alpha, \alpha^{\prime}}{\longrightarrow}\left(\Omega_{X}^{n} \sum_{X}^{n+i-k-1} S_{X} Y\right) \wedge_{X}\left(\Omega_{X}^{n^{\prime}} \sum_{X}^{n^{\prime}+i^{\prime}-k^{\prime}-1} S_{X} Y^{\prime}\right) \\
\longrightarrow \Omega_{X}^{n+n^{\prime}} \sum_{X}^{n+n^{\prime}+i+i^{\prime}-k-k^{\prime}-2}\left(S_{X} Y \wedge_{X} S_{X} Y^{\prime}\right)
\end{gathered}
$$

where we identify $S_{X} Y \wedge_{X} S_{X} Y^{\prime}$ with $S_{X}\left(Y *_{X} Y^{\prime}\right)$ in the obvious manner. 
The reader may verify that the cup product is well-defined, bilinear, and associative.

Thom isomorphism. Let $p: Y \rightarrow X$ be a weak $k$-sphere fibration, and let $\pi: D(p) \rightarrow X$ be the associated weak $(k+1)$-disc fibration; i.e., $D(p)$ is the mapping cylinder of $p$, and $\pi$ is the collapse map. Let $T(p)=D(p) / Y$, the Thom space of $p$.

Let $U_{p} \in \pi_{p}^{k+1}(D(p), Y)$, the universal Thom class of $p$, be the element represented by the composition

$$
D(p) \stackrel{\Delta}{\longrightarrow} D(p) \wedge_{X} D(p) \stackrel{1 \times c}{\longrightarrow} D(p) \wedge_{X} S_{X} Y
$$

where $c$ is the quotient map which collapses $Y \subset D(p)$ to the South polar section in $S_{X} Y$. By a slight abuse of notation, we let $U_{p} \in \pi_{p}^{k+1}(X, Y)$. The next remark follows directly from the definitions [10]:

THEOREM 1.2. If $(X, A)$ is a $C$.W. pair and if $h: A \rightarrow Y$ is a partial section of $p$, then $i * U_{p}=\Gamma(p: h) \in \pi_{p}^{k+1}(X, A)$, the single obstruction to extending $h$ to a full section of $p:$ where $i:(X, A) \rightarrow(D(p), Y)$ is any map such that $i \mid A=h$ and $\pi: i=1_{X}$.

Now let $p^{\prime}: Y^{\prime} \rightarrow X$ be a weak $k^{\prime}$ sphere fibration. We then immediately have (with the obvious notational abuses):

THEOREM 1.3. $U_{p} \cup U_{p^{\prime}}=U_{p \oplus p^{\prime}} \in \pi_{p \oplus p^{\prime}}^{k+k^{\prime}+2}\left(X, Y *_{X} Y^{\prime}\right)$

Finally:

Theorem 1.4 (Thom isomorphism). Let $(X, A)$ be a f.d. C.W. pair, let $p^{\prime}: Y \rightarrow X$ be a weak $k$-sphere fibration over $X$, and let $p$ be any other weak sphere fibration over $X$. Then

$$
\cup U_{p^{\prime}}: \pi_{p}^{\prime}(X, A) \rightarrow \pi_{p \oplus p^{\prime}}^{i+k+1}(X, A \cup Y)
$$

is an isomorphism (where $(X, A \cup Y)$ denotes $\left(D\left(p^{\prime}\right), \pi^{-1} A \cup Y\right)$ ).

We omit the proof, an easy generalization of the proof of Becker's Thom isomorphism [4, Th. 12.8].

2. Obstructions to embedding. Let $M^{m}$ be any compact differentiable manifold, and let $M^{*}$ be the reduced deleted product of $M$, that is, $M^{*}=\left(M \times M-\Delta_{M}\right) / T$, where $T$ exchanges coordinates. Let $P(M)$ be the total space of the projective $(m-1)$-bundle associated with 
the tangent bundle, and let $J: P(M) \rightarrow M^{*}$ be the inclusion defined by $J[v]=[\exp (v), \exp (-v)]$ for any unit tangent vector $v$ of $M$, where exp is the exponential function associated with a suitable metric on $M$. By an abuse of notation, we shall write $P(M) \subset M^{*}$. Let $h$ be the line bundle over $M^{*}$ associated with $T$, let also $h=h \mid P(M)$, and let $L$ be the canonical line bundle over $P^{\infty}$. The inclusion $P^{n-1} \subset P^{\infty}$ can be replaced, up to homotopy, by the $S^{n-1}$ bundle over $P$ associated with $n L$. If $\bar{h}$ classifies $h$, we have a diagram:

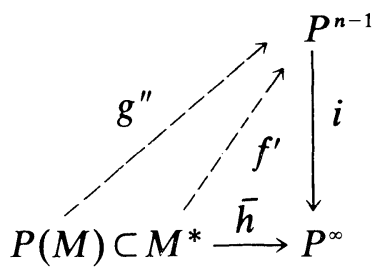

If $f: M \rightarrow R^{n} \quad$ is any embedding, let $f^{\prime}[x, y]=$ $\left[\|f(x)-f(y)\|^{-1}(f(x)-f(y))\right]$ for all $[x, y] \in M^{*}$. Since $i \circ f^{\prime}$ is homotopic to $\bar{h}, n h$ has a section over $M^{*}$ if $M$ embeds in $R^{n}$. If $g: M \rightarrow R^{n}$ is an immersion, let $g^{\prime \prime}$ be the composition $P(M) \stackrel{8_{*}^{*}}{\longrightarrow} P\left(R^{n}\right)=$ $R^{n} \times P^{n-1} \rightarrow P^{n-1}$. Since $i \circ g^{\prime \prime}$ is homotopic to $\bar{h} \mid P(M)$, we have that if $M$ immerses in $R^{n}$, $n h$ has a section over $P(M)$.

Heafliger [6] has shown that $M$ embeds in $R^{n}$ if and only if $n h$ has a section over $M^{*}$, provided $2 n \geqq 3(m+1)$. Furthermore, if $2 n>3(n+1)$, there is a one-to-one correspondence of $\left[M \subset R^{n}\right]$ with the set of homotopy classes of sections of $n h$. Similarly [7], if $2 n \geqq 3 m+1, M$ immerses. in $R^{n}$ if and only if $n h$ has a section over $P(M)$, while if $2 n>3 m+1$, these sections are in one-to-one correspondence with the elements of $\left[M \subseteq R^{n}\right]$. If $g: M \rightarrow R^{n}$ is a fixed immersion, then $g$ is regularly homotopic to an embedding if and only if $g$ " can be extended over all of $M^{*}$, provided $2 n \geqq 3(m+1)$, while if $2 n>3(m+1)$, there is a one-to-one correspondence between rel $P(M)$ homotopy classes of extensions of $g^{\prime \prime}$ and $\pi_{1}\left(\operatorname{Em}\left(M, R^{n}\right), \operatorname{Im}\left(M, R^{n}\right), g\right)$, where $\operatorname{Em}\left(M, R^{n}\right)$ and $\operatorname{Im}\left(M, R^{n}\right)$ are the spaces of embeddings and immersions, respectively.

Now let $\gamma^{n}(M) \in \pi_{n h}^{n}\left(M^{*}\right)$ be the single obstruction to embedding $M$ in $R^{n}$, which we define to be the single obstruction to section of $n h$ over $M^{*}$. By Theorem 1.3. $\gamma^{n}(M)=\left(\gamma^{1}(M)\right)^{n}$. Let $\zeta^{n}(M) \in \pi_{n h}^{n}(P(M))$ be the single obstruction to immersion of $M$ in $R^{n}$, which we define to be the single obstruction to section of $n h$ over $P(M)$. Similarly, $\zeta^{n}(M)=$ $\left(\zeta^{1}(M)\right)^{n}$. If $g: M \rightarrow R^{n}$ is a fixed immersion, let $\gamma^{n}(M, g) \in$ $\pi_{n h}^{n}\left(M^{*}, P(M)\right)$ be the single obstruction to regular homotopy of $g$ to an embedding, defined to be the obstruction to extending $g$ " to a section of $n h$ over $M^{*}$. Now $g$ may also be considered to be an immersion of $M$ into $R^{n+1}$, and by $1.3, \gamma^{n+1}(M, g)=\gamma^{1}(M) \cup \gamma^{n}(M, g)$. 
If $f_{0}, f_{1}: M \rightarrow R^{n}$ are embeddings, let $\delta\left(f_{0}, f_{1}\right) \in \pi_{n h}^{n-1}\left(M^{*}\right)$ be the single difference class, the obstruction to isotopy of $f_{0}$ and $f_{1}$. Specifically, $\delta\left(f_{0}, f_{1}\right)$ can be defined to be the obstruction to homotopy of the sections $f_{0}^{\prime}$ and $f_{1}^{\prime}$ of $n h$ over $M^{*}$ [10]. If, in addition, $f_{0}=g_{0}$ and $f_{1}=g_{1}$, where $g_{t}: M \rightarrow R^{n}, \quad 0 \leqq t \leqq 1$, is a regular homotopy, let $\delta\left(f_{0}, f_{1} ; g_{t}\right) \in$ $\pi_{n h}^{n-1}\left(M^{*}, P(M)\right)$ be the obstruction to regular homotopy of $\left\{g_{t}\right\}$, rel $f_{0}$ and $f_{1}$, to an isotopy. If $g_{0}$ and $g_{1}$ are any immersions, let $\epsilon\left(g_{0}, g_{1}\right) \in$ $\pi_{n h}^{n-1}(P(M))$ be the obstruction to regular homotopy of $g_{0}$ with $g_{1}$, defined to be the obstruction to homotopy of the sections $g_{0}^{\prime \prime}$ and $g_{1}^{\prime \prime}$ of $n h$ over $P(M)[10]$.

3. The obstruction sequence. Affine groups. A set $A$ is said to be an affine group if, for every $a \in A$, an operation ${ }_{a}$ is defined on $A$ such that $\left(A,{ }_{a}\right)$ is a group with identity $a$, and if, for all $a, b, x, y \in A$, $x{ }_{b} y=x b^{-1} y$, where the product and inverse on the right side of the equation are taken with respect to ${ }_{a}$. Note that $\left(A,{ }_{a}\right)$ is then isomorphic to $\left(A,{ }_{b}\right)$ by $x \mapsto x \cdot{ }_{a} b$. Note that every group is an affine group, by $x \cdot{ }_{a} y=x a^{-1} y$.

If $\left(A,{ }_{a}\right)$ is Abelian, we write ${ }_{a}$ for the operation, and we say that $A$ is an Abelian affine group. In that case, let $A^{0}=A \times A / \sim$, the difference group of $A$ (defined only if $A \neq \varnothing$; where $(x, y) \sim(u, v)$ if and only if $\left.x+{ }_{y} v=u\right)$, an Abelian group isomorphic to each $\left(A,+_{a}\right)$, by $[x, a] \mapsto x$.

Becker [3] has shown that in the metastable range, i.e., $2 n>$ $3(m+1),\left[M \subset R^{n}\right]$ is an Abelian affine group which, if nonempty, has difference group isomorphic to $\pi_{n h}^{n-1}\left(M^{*}\right)$, by $\left[\left[f_{1}\right],\left[f_{2}\right]\right] \mapsto \delta\left(f_{1}, f_{2}\right)$. If $f_{1}, f_{2}, f_{3}, f_{4}$ are embeddings of $M$ in $R^{n},\left[f_{1}\right]+_{\left[f_{2}\right]}\left[f_{3}\right]=\left[f_{4}\right]$ if and only if $\delta\left(f_{1}, f_{2}\right)=\delta\left(f_{4}, f_{3}\right)$. Thus $\left[M \subset R^{n}\right]$, if nonempty, is noncanonically isomorphic (as an affine group) to $\pi_{n h}^{n-1}\left(M^{*}\right)$.

Similarly, in the metastable range, $2 n>3 m+1$ in that case, $\left[M \subseteq R^{n}\right]$ is an Abelian affine group which, if nonempty, has difference group $\pi_{n h}^{n-1}(P(M))$, while if $2 n>3(m+1)$ and $g$ is an immersion, $\pi_{1}\left(\operatorname{Im}\left(M, R^{n}\right), \operatorname{Em}\left(M, R^{n}\right), g\right)$ is an Abelian affine group with difference group $\pi_{n h}^{n-1}\left(M^{*}, P(M)\right)$.

Since twisted cohomotopy is a twisted cohomology theory, we have an exact sequence

$$
\pi_{n h}^{n-1}\left(M^{*}\right) \stackrel{J *}{\longrightarrow} \pi_{n h}^{n-1}(P(M)) \stackrel{\delta}{\longrightarrow} \pi_{n h}^{n}\left(M^{*}, P(M)\right)
$$

$$
\stackrel{K^{*}}{\longrightarrow} \pi_{n h}^{n}\left(M^{*}\right) \stackrel{J^{*}}{\longrightarrow} \pi_{n h}^{n}(P(M)) .
$$

Now we say that a sequence $A_{1} \stackrel{\alpha_{1}}{\rightarrow} A_{2} \stackrel{\alpha_{2}}{\rightarrow} A_{3}$ of Abelian affine groups and morphisms is exact at $A_{2}$ if either $A_{1}$ is empty or there exists $a \in A_{3}$ such that $\alpha_{1}\left(A_{1}\right)=\alpha_{2}^{-1} a$. If $2 n>3(m+1)$, we have a sequence of Abelian affine groups and morphisms 


$$
\begin{gathered}
{\left[M \subset R^{n}\right] \stackrel{\phi_{n}}{\longrightarrow}\left[M \subseteq R^{n}\right] \stackrel{\kappa}{\longrightarrow} \pi_{n h}^{n}\left(M^{*}, P(M)\right)} \\
\stackrel{K^{*}}{\longrightarrow} \pi_{n h}^{n}\left(M^{*}\right) \stackrel{J^{*}}{\longrightarrow} \pi_{n h}^{n}(P(M))
\end{gathered}
$$

where $\phi_{n}$ sends each isotopy class to the regular homotopy class containing it; and where, if $g$ is an immersion, $\kappa[g]=\gamma^{n}(M ; g)$. Now (3.2) is exact, as the reader can easily verify, and the sequence of difference groups of the nonempty portion of (3.2) is the corresponding portion of (3.1).

We also have a commutative diagram of Abelian groups and homomorphisms

$$
\begin{array}{r}
\pi_{(n+1) h}^{n}\left(M^{*}\right) \stackrel{J^{*}}{\longrightarrow} \pi_{(n+1) h}^{n}(P(M)) \\
\prod_{\pi_{n h}^{n-1}\left(M^{*}\right) \stackrel{J^{*}}{\longrightarrow}}^{\longrightarrow} \pi_{n h}^{n-1}(P(M))
\end{array}
$$

and a commutative diagram of Abelian affine groups and morphisms

$$
\begin{gathered}
{\left[M \subset R^{n+1}\right] \stackrel{\phi_{n+1}}{\longrightarrow}\left[M \subseteq R^{n+1}\right]} \\
\uparrow \mathscr{E}_{n} \\
{\left[M \subset R^{n}\right] \stackrel{\phi_{n}}{\longrightarrow}\left[M \subseteq \mathscr{I}_{n}\right.}
\end{gathered}
$$

The relationship between diagrams (3.3) and (3.4) is as follows: the diagram of difference groups of the nonempty portion of (3.4) is the corresponding portion of (3.3). Thus, if $\left[M \subset R^{n}\right]$ is nonempty, and if a specific embedding $f$ is chosen, the two diagrams can be identified.

4. Calculation of the groups. In $\S 3$, we observed that computation of diagram (0.1) reduced to computation of the following diagram, provided $P^{m}$ embeds in $R^{2 m-2}$.

$$
\begin{array}{ccc}
\pi_{2 m h}^{2 m-1}\left(\left(P^{m}\right)^{*}\right) \stackrel{J^{*}}{\longrightarrow} \pi_{2 m h}^{2 m-1}\left(P\left(P^{m}\right)\right) & \uparrow \cup \zeta^{1}\left(P^{m}\right) \\
\uparrow \cup \gamma^{1}\left(P^{m}\right) & & \\
\pi_{(2 m-1) h}^{2 m-2}\left(\left(P^{m}\right)^{*}\right) \stackrel{J^{*}}{\longrightarrow} \pi_{(2 m-1) h}^{2 m-2}\left(P\left(P^{m}\right)\right) \\
\uparrow \cup \gamma^{1}\left(P^{m}\right) & \uparrow \cup \zeta^{1}\left(P^{m}\right) \\
\pi_{(2 m-2) h}^{2 m-3}\left(\left(P^{m}\right)^{*}\right) \stackrel{J^{*}}{\longrightarrow} \pi_{(2 m-2) h}^{2 m-3}\left(P\left(P^{m}\right)\right) .
\end{array}
$$

In this section we compute the groups in (4.1); in succeeding sections, we compute the maps.

Recall from $[3, \S 4]$ or $[10, \S 5]$ that if $\xi$ is a vector bundle over $X$ 
where $(X, A)$ is an $n$-dimensional C.W. pair, the Atiyah-Hirzebruch spectral sequence for $\pi_{\xi}^{*}(X, A)$ consists of filtrations

$$
\pi_{\xi}^{i}(X, A)=F^{0} \pi_{\xi}^{i}(X, A) \supset F^{1} \pi_{\xi}^{i}(X, A) \supset \cdots \supset F^{n-i+1} \pi_{\xi}^{i}(X, A)=0
$$

and canonical isomorphisms

$$
d_{i}^{s+\imath}: F^{s} \pi_{\xi}^{i}(X, A) / F^{s+1} \pi_{\xi}^{i}(X, A) \rightarrow D^{s} \pi_{\xi}^{i}(X, A) / C^{s} \pi_{\xi}^{i}(X, A)
$$

where $C^{s} \pi_{\xi}^{i}(X, A) \subset D^{s} \pi_{\xi}^{i}(X, A) \subset H^{i+s}\left(X, A ; \pi_{s} \otimes \Gamma_{\xi}\right)$. Here, $\pi_{s}$ is the $s$ th stable homotopy group of the 0 -sphere and $\Gamma_{\xi}$ is the local system of integers determined by $\xi$. From now on, we identify $F^{s} \pi_{\xi}^{i}(X, A) / F^{s+1} \pi_{\xi}^{i}(X, A)$ with $D^{s} \pi_{\xi}^{i}(X, A) / C^{s} \pi_{\xi}^{i}(X, A)$, and omit " $(X, A)$ " from the notation when no confusion can arise.

In the cases of interest to us, the cohomology dimension of $X$ is $2 m-1$ and $i \geqq 2 m-3$. In this case, the descriptions of $C^{s} \pi_{\xi}^{i}$ and $D^{s} \pi_{\xi}^{i}$ are well-known.

Define homomorphisms $\alpha_{i}(\xi): H^{*}\left(X, A ; Z_{2}\right) \rightarrow H^{*}\left(X, A ; Z_{2}\right)$, for $i=1,2, \quad$ by $\quad \alpha_{1}(\xi)(x)=S q^{1} x+x \cup w_{1} \xi, \quad$ and $\quad \alpha_{2}(\xi)(x)=S q^{2} x+$ $S q^{1} x \cup w_{1} \xi+x \cup\left(w_{1} \xi\right)^{2}+x \cup w_{2} \xi$. The homomorphisms $\alpha_{1}(\xi)$ and $\alpha_{2}(\xi)$ for all vector bundles $\xi$ define cohomology operations $\alpha_{1}(B O)$ and $\alpha_{2}(B O)$ in the category of spaces over $B O$ (see [14]).

Let $\rho: H^{*}\left(X, A ; \Gamma_{\xi}\right) \rightarrow H^{*}\left(X, A ; Z_{2}\right)$ be reduction $\bmod 2$, and $\Phi_{2}(B O)$ be the twisted stable secondary cohomology operation (in the category of spaces over $B O)$ with the relation $\alpha_{2}(B O) \circ\left(\alpha_{2}(B O) \circ \rho\right)=0$, which arises from the first three stages of the standard Postnikov factorization of the sphere bundle of a universal $n$-vector bundle with $n$ large. Write

$$
\Phi_{2}(\xi): \operatorname{ker}\left(\alpha_{2}(\xi) \circ \rho\right) \rightarrow H^{*}\left(X, A ; Z_{2}\right) / \operatorname{Im}\left(\alpha_{2}(\xi)\right)
$$

Proposition 4.1. Assume $(X, A)$ has relative äimension $\leqq i+2$. Then

(1) $C^{0} \pi_{\xi}^{i}=0, D^{0} \pi_{\xi}^{i}=\operatorname{ker}\left(\alpha_{2}(\xi) \circ \rho\right) \subset H^{i}\left(X, A ; \Gamma_{\xi}\right)$

(2) $\left.C^{1} \pi_{\xi}^{l}=\alpha_{2}(\xi) \rho H^{i-1}(X, A) ; \Gamma_{\xi}\right), D^{1} \pi_{\xi}^{i}=H^{i+1}\left(X, A ; Z_{2}\right)$

(3) $C^{2} \pi_{\xi}^{i}=\operatorname{Im}\left(\Phi_{2}(\xi) \subset H^{i+2}\left(X, A ; Z_{2}\right), D^{2} \pi_{\xi}^{i}=H^{i+2}\left(X, A ; Z_{2}\right)\right.$

(4) $D^{\prime} \pi_{\xi}^{i}=C^{j} \pi_{\xi}^{i}=0$ for $j>i+2$.

Proposition 4.1 can be proved by the same technique that McClendon used to prove part 4 of Theorem 6.1 in [15]. Now since $\Phi_{2}(\xi)$ has the relation $\alpha_{2}(B O) \circ\left(\alpha_{2}(B O) \rho\right)=0$, it follows immediately that

REMARK 4.2. $C^{2} \pi_{\xi}^{\iota} \supset \alpha_{2}(\xi) H^{i}\left(X, A ; Z_{2}\right)$. 
When $\operatorname{dim}(X, A) \leqq i+2,4.1$ allows for the calculation of $\pi_{\xi}^{i}$ up to extension. The following proposition allows for the calculation of the extensions in many cases (and in particular for the cases in this paper). Let $R_{2}$ be the set of elements of $F^{0} \pi_{\xi}^{i} / F^{1} \pi_{\xi}^{i}$ of order 2 , and define

$$
\begin{aligned}
& \theta_{1}: R_{2} \rightarrow F^{1} \pi_{\xi}^{i} / F^{2} \pi_{\xi}^{i} \\
& \theta_{2}: F^{1} \pi_{\xi}^{i} / F^{2} \pi_{\xi}^{i} \rightarrow F^{2} \pi_{\xi}^{i}
\end{aligned}
$$

as follows: if $z \in F^{0} \pi_{\xi}^{i} / F^{1} \pi_{\xi}^{i}$ and $2 z=0$, let $x \in F^{0} \pi_{\xi}^{i}$ which represents $z$. Then $\theta_{1}(z)$ is represented by $2 x$. If $z \in F^{1} \pi_{\xi}^{i} / F^{2} \pi_{\xi}^{i}$ and $x \in F^{1} \pi_{\xi}^{i}$ represents $z$, let $\theta_{2}(z)=2 x$.

Let $\delta(\xi): H^{*}\left(X, A ; Z_{2}\right) \rightarrow H^{*}\left(X, A ; \Gamma_{\xi}\right)$ be the Bockstein of the short exact sequence $\Gamma_{\xi} \stackrel{\times 2}{\rightarrow} \Gamma_{\xi} \rightarrow Z_{2}$ of coefficients. Note that $\rho \delta(\xi)=$ $\alpha_{1}(\xi)$.

Proposition 4.3 (Larmore-Thomas [12]). Identify $F^{s} \pi_{\xi}^{i} / F^{s+1} \pi_{\xi}^{i}$ with $D^{s} \pi_{\xi}^{i} / C^{s} \pi_{\xi}^{i}$. (1) For any $z \in R_{2}, \quad \theta_{1}(z)=\alpha_{2}(\xi)\left(\delta(\xi)^{-1}(z)\right)$. (2) If $z \in D^{1} \pi_{\xi}^{i} / C^{1} \pi_{\xi}^{i}$, let $x \in D^{1} \pi_{\xi}^{i}$ represent $z$. Then $\theta_{2}(z)=\alpha_{1}(\xi) x+C^{2} \pi_{\xi}^{i}$.

To calculate the groups in diagram (4.1), we need the following descriptions of the $Z_{2}$-cohomology rings of $P\left(P^{m}\right)$ and $\left(P^{m}\right)^{*}$.

Proposition 4.4 (see [9, Ch. 16]). Let $v$ be the first Stiefel-Whitney class of the canonical line bundle over $P\left(P^{m}\right)$ and let $q: P\left(P^{m}\right) \rightarrow P^{m}$ be the bundle projection. Then $q^{*}$ is injective on $H^{*}\left(P^{m} ; Z_{2}\right)$, and, as an $H^{*}\left(P^{m} ; Z_{2}\right)$ module, $H^{*}\left(P\left(P^{m}\right) ; Z_{2}\right)$ has $1, v, v^{2}, \cdots, v^{m-1}$ as basis. Moreover, $v^{m}=\sum_{i=1}^{m}\left(w_{i} P^{m}\right) v^{m-i}$, where $w_{i} P^{m}$ is the ith Stiefel-Whitney class of $P^{m}$.

Proposition 4.5 (Handel [8]). Let $u \in H^{1}\left(\left(P^{m}\right)^{*} ; Z_{2}\right)$ be the first Stiefel-Whitney class of the 0-sphere bundle $P^{m} \times P^{m}-\Delta P^{m} \rightarrow\left(P^{m}\right)^{*}$. Then

(1) $\left(P^{m}\right)^{*}$ has the homotopy type of a closed $(2 m-1)$-manifold;

(2) There are elements $y_{1} \in H^{1}\left(\left(P^{m}\right)^{*} ; Z_{2}\right), y_{2} \in H^{2}\left(\left(P^{m}\right)^{*} ; Z_{2}\right)$ such that $H^{*}\left(\left(P^{m}\right)^{*} ; Z_{2}\right)=Z_{2}\left[u, y_{1}, y_{2}\right] / \mathscr{A}$ where $\mathscr{A}$ is the ideal generated by $u^{2}-u y_{1}, b_{m}$, and $b_{m+1} ;$ where $b_{1}=\sum_{j=0}^{[1 / 2 i]}\left(\begin{array}{c}i-j \\ j\end{array}\right) y_{1}^{i-2 j} y_{2}^{j}$;

(3) $S q^{1} y_{2}=y_{1} y_{2}$;

(4) Let $m=2^{r}+s, 0 \leqq s<2^{r}$. Then $y_{1}^{2^{r+1}-1}=0$

and $u y_{1}^{2^{r+1}-2} y_{2}^{s}$ generates $H^{2 m-1}\left(\left(P^{m}\right)^{*} ; Z_{2}\right)$. 
We also need the following lemma. Let $Z[a]$ be the local system of integers over a space $X$, twisted by $a \in H^{1}\left(X ; Z_{2}\right)$. Define $\alpha: H^{*}\left(X, A ; Z_{2}\right) \rightarrow H^{*}\left(X, A ; Z_{2}\right)$ by $\alpha(x)=S q^{1} x+x \cup a$.

LemMa 4.6. Assume $H^{i}(X, A ; Z[a])$ is finitely generated. Let $r(i)$ be the dimension of $\alpha H^{i-1}\left(X, A ; Z_{2}\right)$ and $s(i)$ the dimension of $\operatorname{ker} \alpha \subset$ $H^{i}\left(X, A ; Z_{2}\right)$ (considered as vector spaces over $\left.Z_{2}\right)$. Then $H^{i}(X, A ; Z[a]) \cong G \bigoplus H$ where $G$ is the direct sum of $r(i)$ copies of $Z_{2}$ and $H \otimes Z_{2}$ has dimension $\leqq s(i)-r(i)$.

The proof is elementary and will be left to the reader. The reader can also easily verify that $H^{i}\left(\left(P^{m}\right)^{*} ; Z\right), H^{i}\left(P\left(P^{m}\right) ; Z\right), H^{i}\left(\left(P^{m}\right)^{*} ; \Gamma_{h}\right)$, and $H^{i}\left(P\left(P^{m}\right) ; \Gamma_{h}\right)$ are 2-primary groups for $m<i<2 m-1$.

Most of the calculations of the groups in diagram (4.1) are direct using 4.1 through 4.6 ; a few require some ingenuity. We indicate the details in the case $m \equiv 3 \bmod 4$ only. In that case, we have (where $\left.m=2^{r}+s, 0 \leqq s<2^{r}\right)$ :

$$
\begin{array}{lll}
H^{2 m-1}\left(\left(P^{m}\right)^{*} ; Z_{2}\right) \cong Z_{2} & \text { generated by } & u y_{1}^{2^{r+1}-2} y_{2}^{s} \\
H^{2 m-1}\left(\left(P^{m}\right)^{*} ; Z\right) \cong Z_{2} & \text { generated by } & \delta\left(u y_{1}^{2^{r+1}-3} y_{2}^{s}\right) ;
\end{array}
$$$$
H^{2 m-2}\left(\left(P^{m}\right)^{*} ; Z_{2}\right) \cong Z_{2} \oplus Z_{2} \text { generated by } u y_{1}^{2^{r+1}-3} y_{2}^{s} \text { and } y_{1}^{2^{r+1}-2} y_{2}^{s} \text {; }
$$$$
H^{2 m-2}\left(\left(P^{m}\right)^{*} ; \Gamma_{h}\right) \cong Z_{2} \text { generated by } \delta(h)\left(y_{1}^{2^{r+1}-3} y_{2}^{s}\right) \text {; }
$$$$
H^{2 m-3}\left(\left(P^{m}\right)^{*} ; Z\right) \cong Z_{2} \oplus Z_{2} \text { generated by } \delta\left(u y_{1}^{2^{r+1}-5} y_{2}^{s}\right) \text { and } \delta\left(y_{1}^{2^{2+1}-4} y_{2}^{s}\right) \text {; }
$$
$\rho H^{2 m-3}\left(\left(P^{m}\right)^{*} ; \Gamma_{h}\right)$ is generated by $u y_{1}^{2^{r+1}-2} y_{2}^{s-1}$ and $y_{1}^{2^{2+1-3}} y_{2}^{s}+u y_{1}^{2^{r+1}-4} y_{2}^{s}$. $H^{2 m-4}\left(\left(P^{m}\right)^{*} ; Z\right) \quad$ is generated by $\delta\left(u y_{1}^{2^{r+1}-4} y_{2}^{s-1}\right)$ and $\delta\left(y_{1}^{2^{r+1}-3} y_{2}^{s-1}\right)$.

We illustrate the use of $4.4,4.5$, and 4.6 by writing out the calculation of $H^{2 m-2}\left(\left(P^{m}\right)^{*} ; \Gamma_{h}\right)$ and $H^{2 m-3}\left(\left(P^{m}\right)^{*} ; Z\right)$. First observe that $H^{2 m-2}\left(\left(P^{m}\right)^{*} ; Z_{2}\right) \cong H^{1}\left(\left(P^{m}\right)^{*} ; Z_{2}\right) \cong Z_{2} \oplus Z_{2}$ and so $u y_{1}^{2^{r+1}-3} y_{2}^{s}$ and $y_{1}^{2^{r+1}-2} y_{2}^{s}$ generate $H^{2 m-2}\left(\left(P^{m}\right)^{*} ; Z_{2}\right)$. Since

$$
\begin{aligned}
& \alpha_{1}(h)\left(y_{1}^{2^{r+1}-2} y_{2}^{s}\right)=u y_{1}^{2^{r+1}-2} y_{2}^{s} \neq 0 \\
& \alpha_{1}(h)\left(y_{1}^{2 r+1-3} y_{2}^{s}\right)=u y_{1}^{2^{r+1}-3} y_{2}^{s},
\end{aligned}
$$

Lemma 4.6 implies that $\delta(h)\left(y_{1}^{2^{r+1-3}} y_{2}^{s}\right)$ generates $H^{2 m-2}\left(\left(P^{m}\right)^{*} ; \Gamma_{h}\right)$. Since $S q^{1}\left(u y_{1}^{2 r+1}-5 y_{2}^{s}\right)=u y_{1}^{2^{r+1}-4} y_{2}^{s}$ and $S q^{1}\left(y_{1}^{2^{r+1}-4} y_{2}^{s}\right)=y_{1}^{2^{r+1}-3} y_{2}^{s}$, we have that $\delta\left(u y_{1}^{2^{r+1}-5} y_{2}^{s}\right)$ and $\delta\left(y_{1}^{2^{r+1}-4} y_{2}^{s}\right)$ generate a subgroup of $H^{2 m-3}\left(\left(P^{m}\right)^{*} ; Z\right)$ isomorphic to $Z_{2} \oplus Z_{2}$. But $H^{2 m-3}\left(\left(P^{m}\right)^{*} ; Z\right)$ must by isomorphic to $Z_{2} \oplus Z_{2}$ since 


$$
\begin{aligned}
Z_{2} \oplus Z_{2} \oplus Z_{2} & \cong H^{2 m-3}\left(\left(P^{m}\right)^{*} ; Z_{2}\right) \\
& =\left(H^{2 m-3}\left(\left(P^{m}\right)^{*} ; Z\right) \otimes Z_{2}\right) \oplus\left(H^{2 m-2}\left(\left(P^{m}\right)^{*} ; Z\right) * Z_{2}\right)
\end{aligned}
$$

and

$$
H^{2 m-2}\left(\left(P^{m}\right)^{*} ; Z\right) * Z_{2}
$$

is isomorphic to $Z_{2}$, where $*$ is the torsion product.

Calculation of $\pi_{2 m h}^{2 m-1}\left(\left(P^{m}\right)^{*}\right)$ : By Proposition (4.1),

$$
\pi_{2 m h}^{2 m-1}\left(\left(P^{m}\right)^{*}\right) \cong H^{2 m-1}\left(\left(P^{m}\right)^{*} ; Z\right) \cong Z_{2}
$$

Calculation of $\pi_{(2 m-1) h}^{2 m-2}\left(\left(P^{m}\right)^{*}\right)$ :

$$
\begin{aligned}
& \alpha_{2}((2 m-1) h)\left(u y_{1}^{2^{+1}-2} y_{2}^{s-1}\right)=0 . \\
& \alpha_{2}((2 m-1) h)\left(y_{1}^{2^{2+1}-3} y_{2}^{s}+u y_{1}^{2^{r+1}-4} y_{2}^{s}\right)=u y_{1}^{2^{r+1}-4} y_{2}^{s+1} .
\end{aligned}
$$

LEMMA 4.7. If $s$ is odd, $y_{1}^{2^{r+1}-4} y_{2}^{s+1}=0$.

Proof. It is clear from 4.5 that if $y_{1}^{2^{r+1}-4} y_{2}^{s+1} \neq 0$, it must equal $y_{1}^{2^{r+1}-2} y_{2}^{s}$. But in the next section (Proposition 5.3), we shall see that $J^{*}(u)=J^{*}\left(y_{1}\right)=v$ and $J^{*}\left(y_{2}\right)=z^{2}+z v$ where $z$ is the generator of $H^{1}\left(P\left(P^{m}\right) ; Z_{2}\right)$. Hence by 4.4

$$
\begin{aligned}
& J^{*}\left(y_{1}^{2^{r+1}-4} y_{2}^{s+1}\right)=v^{2^{r+1}-4}\left(z^{2}+z v\right)^{s+1}=0 \\
& J^{*}\left(y_{1}^{2^{r+1}-2} y_{2}^{s}\right)=v^{2^{r+1}-2}\left(z^{2}+z v\right)^{s} \neq 0 .
\end{aligned}
$$

Thus $y_{1}^{2^{r+1}-4} y_{2}^{s+1}$ must be 0 .

Applying Proposition 4.1 again, we have then

$$
\begin{array}{lll}
D^{0} \pi_{(2 m-1) h}^{2 m-2}\left(\left(P^{m}\right)^{*}\right) \cong Z_{2} & \text { generated by } & \delta(h)\left(y_{1}^{2^{r+1}-3} y_{2}^{s}\right) \\
D^{1} \pi_{(2 m-1) h}^{2 m-2}\left(\left(P^{m}\right)^{*}\right) \cong Z_{2} & \text { generated by } & u y_{1}^{2^{r+1}-2} y_{2}^{s} \\
C^{1} \pi_{(2 m-1) h}^{2 m-2}\left(\left(P^{m}\right)^{*}\right)=0 . &
\end{array}
$$

The extension is nontrivial since

$$
\alpha_{2}((2 m-1) h)\left(y_{1}^{2^{r+1}-3} y_{2}^{s}\right)=u y_{1}^{2 r+1-2} y_{2}^{s}+y_{1}^{2^{r+1}-3} y_{2}^{s+1}=u y_{1}^{2^{r+1}-2} y_{2}^{s}
$$

which implies that $\theta_{1}$ of Proposition 4.3 is nontrivial.

Calculation of $\pi_{(2 m-2) h}^{2 m-3}\left(\left(P^{m}\right)^{*}\right)$ :

$D^{0} \pi_{(2 m-2) h}^{2 m-3}\left(\left(P^{m}\right)^{*}\right) \cong Z_{2} \oplus Z_{2}$ generated by $\delta\left(u y_{1}^{2^{r+1}-5} y_{2}^{s}\right)$ and $\delta\left(y_{1}^{2^{2+1}-4} y_{2}^{s}\right) ;$ 


$$
\begin{aligned}
& D^{1} \pi_{(2 m-2) h}^{2 m-3}\left(\left(P^{m}\right)^{*}\right) \cong Z_{2} \oplus Z_{2} \text { generated by } u y_{1}^{r^{r+1}-3} y_{2}^{s} \text { and } y_{1}^{2^{r+1}-2} y_{2}^{s} \\
& C^{1} \pi_{(2 m-2) h}^{2 m-3}\left(\left(P^{m}\right)^{*}\right)=0 ; \\
& D^{2} \pi_{(2 m-2) h}^{2 m-3}\left(\left(P^{m}\right)^{*}\right) \cong Z_{2} \text { generated by } u y_{1}^{2^{r+1}-2} y_{2}^{s} \\
& C^{2} \pi_{(2 m-2) h}^{2 m-3}\left(\left(P^{m}\right)^{*}\right)=0 .
\end{aligned}
$$

The computations of these groups are all direct with the exception of that of $C^{2} \pi_{(2 m-2) h}^{2 m-3}\left(\left(P^{m}\right)^{*}\right)$ which is given at the end of this section. All of the extensions are nontrivial as is shown by direct calculation of $\theta_{1}$ and $\theta_{2}$ using Proposition 4.3.

In a similar manner, we obtain:

$$
\begin{aligned}
& \pi_{2 m h}^{2 m-1}\left(P\left(P^{m}\right)\right) \cong Z_{2} ; \\
& D^{0} \pi_{(2 m-1) h}^{2 m-2}\left(P\left(P^{m}\right)\right) \cong Z_{2} \oplus Z_{2} \quad \text { generated by } \delta(h)\left(z^{m} v^{m-3}\right)
\end{aligned}
$$

and $\delta(h)\left(z^{m-2} v^{m-1}\right)$ where $z$ is the generator of $\left.H^{1}\left(P\left(P^{m}\right) ; Z_{2}\right)\right)$;

$D^{1} \pi_{(2 m-1) h}^{2 m-2}\left(P\left(P^{m}\right)\right) \cong Z_{2} \quad$ generated by $\quad z^{m} v^{m-1}$

$C^{1} \pi_{(2 m-1) h}^{2 m-2}\left(P\left(P^{m}\right)\right)=0$;

$D^{0} \pi_{(2 m-2) h}^{2 m-3}\left(P\left(P^{m}\right)\right) \cong Z_{2} \oplus Z_{2}$ generated by $\delta\left(z^{m} v^{m-4}\right)$ and $\delta\left(z^{m-2} v^{m-2}\right)$;

$D^{1} \pi_{(2 m-2) h}^{2 m-3}\left(P\left(P^{m}\right)\right) \cong Z_{2} \oplus Z_{2} \quad$ generated by $z^{m-1} v^{m-1}$ and $z^{m} v^{m-2}$;

$C^{1} \pi_{(2 m-2) h}^{2 m-3}\left(P\left(P^{m}\right)\right)=0$;

$D^{2} \pi_{(2 m-2) h}^{2 m-3}\left(P\left(P^{m}\right)\right) \cong Z_{2} \quad$ generated by $\quad z^{m} v^{m-1}$;

$C^{2} \pi_{(2 m-2) h}^{2 m-3}\left(P\left(P^{m}\right)\right)=0$.

The extensions in $\pi_{(2 m-1) h}^{2 m-2}\left(P\left(P^{m}\right)\right)$ and $\pi_{(2 m-2) h}^{2 m-3}\left(P\left(P^{m}\right)\right)$ are all nontrivial.

The remainder of $\S 4$ is devoted to proving that $C^{2} \pi_{(2 m-2) h}^{2 m-3}\left(\left(P^{m}\right)^{*}\right)=0$. Recall that that group equals the image of $\Phi_{2}((2 m-2) h)$ where $\Phi_{2}((2 m-2) h)$ is twisted by $(2 m-2) h$, or, what is the same thing when thought of as an operation in the category of spaces over $B O$, twisted by a classifying map $f:\left(P^{m}\right)^{*} \rightarrow B O$ for the stable class of $(2 m-2) h$. Since any multiple of $4 h$ is a spin bundle, $f$ factors through BSpin, which is 3-connected. Now the degree of $\Phi_{2}(B O)$ (and its pullback to BSpin) is 3; thus $\Phi_{2}((2 m-2) h)=\Phi_{2}$, where $\Phi_{2}$ is a (non-twisted) secondary cohomology operation with relation $S q^{2} \circ S q^{2}=0$. (See [16, §3].)

Observe that

$$
\begin{gathered}
S q^{2}\left(\rho \delta\left(u y_{1}^{2^{r+1}-4} y_{2}^{s-1}\right)=S q^{2}\left(u y_{1}^{2^{r+1}-3} y_{2}^{s-1}\right)=0\right. \\
S q^{2}\left(\rho \delta\left(y_{1}^{2^{r+1}-3} y_{2}^{s-1}\right)=S q^{2}\left(y_{1}^{r^{r+1}-2} y_{2}^{s-1}\right)=0\right.
\end{gathered}
$$

so that $\Phi_{2}$ is defined on all of $H^{2 m-4}\left(\left(P^{m}\right)^{*} ; Z\right)$. By Handel [8] there is a 
$(2 m-2)$-dimensional space $B G$, a map $k:\left(P^{m}\right)^{*} \rightarrow B G$ and elements $x_{1}, x_{2} \in H^{*}\left(B G ; Z_{2}\right)$ such that $k^{*} x_{i}=y_{i}$ for $i=1,2$. Then

$$
\Phi_{2}\left(\delta\left(y_{1}^{2^{r+1}-3} y_{2}^{s-1}\right)\right)=k^{*} \Phi_{2}\left(\delta\left(x_{1}^{2^{r+1}-3} x_{2}^{s-1}\right)\right)=0
$$

for dimensional reasons.

In order to calculate $\Phi_{2}\left(\delta\left(u y_{1}^{2^{r+1-4}} y_{2}^{s-1}\right)\right)$, we need the following construction which will also be used in $\S 5$. For any space $X$, let $\wedge^{2} X$ be the space of unordered (not necessarily distinct) pairs of elements of $X$, topologized as a quotient space of $X^{2}$. Then $\Delta X \subset \wedge^{2} X$ and $X^{*} \subset \wedge^{2} X$.

For any $1 \leqq \mathscr{I} \leqq m$, let $P_{\mathscr{y}}^{m}=P^{m} / P^{\Phi-1}$. In [11], elements $\wedge z^{i} \in H^{i}\left(\wedge^{2} P_{\mathscr{q}}^{m}, \Delta P_{\mathscr{G}}^{m} ; Z_{2}\right)$ are defined for $\mathscr{I} \leqq i \leqq m$, and an action of $Z_{2}[u]$ on $H^{*}\left(\wedge^{2} P_{g}^{m}, \Delta P_{\mathscr{y}}^{m} ; Z_{2}\right)$ is defined (where $u$ can be regarded as the element in Proposition 4.5). The elements $u^{i} \wedge z^{j}$, for $0 \leqq i \leqq j, \mathscr{I} \leqq j \leqq$ $m$, generate $H^{*}\left(\wedge^{2} P_{g}^{m}, \Delta P_{g}^{m} ; Z_{2}\right)$ as a ring; the relations among the $u^{i} \wedge z^{j}$ are described in [11]. (Note that $u^{i} \wedge z^{j} \in H^{*}\left(\wedge^{2} P_{g}^{m}, \Delta P_{g}^{m} ; Z_{2}\right)$ is mapped to $u^{i} \wedge z^{j} \in H^{*}\left(\wedge^{2} P_{{ }^{-1}-1}^{m}, \Delta P_{9-1}^{m} ; Z_{2}\right)$ by the obvious map. $)$

Now let $j:\left(P^{m}\right)^{*} \rightarrow\left(\wedge^{2} P^{m}, \Delta P^{m}\right)$ be the inclusion.

LEMMA 4.8 .

(1) $j^{*}\left(\wedge z^{m-3} \wedge z^{m-1}\right)=u y_{1}^{2 r+1}-3 y_{2}^{s-1}+y_{1}^{2^{r+1}-2} y_{2}^{s-1}$

(2) $j^{*}\left(\wedge z^{m-3} \wedge z^{m}\right)=u y_{1}^{2^{r+1}-4} y_{2}^{s}+y_{1}^{2^{r+1}-3} y_{2}^{s}$;

(3) $j^{*}\left(\wedge z^{m-2} \wedge z^{m}\right)=u y_{1}^{2^{r+1}-3} y_{2}^{s}+y_{1}^{2^{r+1}-2} y_{2}^{s}$.

We give the proof below; but first we finish the calculation of $C^{2} \pi_{(2 m-2) h}^{2 m-3}\left(\left(P^{m}\right)^{*}\right)$. Let $g:\left(\wedge^{2} P^{m}, \Delta P^{m}\right) \rightarrow\left(\wedge^{2} P_{m-3}^{m}, \Delta P_{m-3}^{m}\right)$ be the natural map. Since $m \equiv 3 \bmod 4, P_{m-3}^{m}$ has the same homotopy type as $P_{m-2}^{m} \vee S^{m-3}$. Let $h:\left(\wedge^{2} P_{m-2}^{m}, \Delta P_{m-2}^{m}\right) \rightarrow\left(\wedge^{2} P_{m-3}^{m}, \Delta P_{m-3}^{m}\right)$ be the map induced by $P_{m-2}^{m} \rightarrow P_{m-2}^{m} \vee S^{m-3} \rightarrow P_{m-3}^{m}$ where the first map is the inclusion and the second is a homotopy equivalence.

Now $S q^{1}\left(\wedge z^{m-3} \wedge z^{m-2}\right)=\wedge z^{m-3} \wedge z^{m-1}$ by Lemma 10 of [11] and

$$
(g j)^{*}\left(\wedge z^{m-3} \wedge z^{m-1}\right)=u y_{1}^{2^{r+1}-3} y_{2}^{s-1}+y_{1}^{2^{r+1}-2} y_{2}^{s-1}=\rho \delta\left(u y_{1}^{2^{r+1}-4} y_{2}^{s-1}+y_{1}^{2^{r+1}-3} y_{2}^{s-1}\right)
$$

by Lemma 4.8. Hence it suffices to show that $\Phi_{2}\left(\delta\left(\wedge z^{m-3} \wedge z^{m-2}\right)\right)=0$ in $H^{2 m-1}\left(\wedge^{2} P_{m-3}^{m}, \Delta P_{m-3}^{m} ; Z_{2}\right)$. But $\Phi_{2}$ is defined on $\delta\left(\wedge z^{m-3} \wedge z^{m-2}\right)$ and we have a commutative diagram

$$
\begin{gathered}
H^{2 m-1}\left(\wedge^{2} P_{m-3}^{m}, \Delta P_{m-3}^{m} ; Z_{2}\right) \stackrel{h^{*}}{\longrightarrow} H^{2 m-1}\left(\wedge^{2} P_{m-2}^{m}, \Delta P_{m-2}^{m} ; Z_{2}\right) \\
\uparrow \Phi_{2} \\
H^{2 m-4}\left(\wedge^{2} P_{m-3}^{m}, \Delta P_{m-3}^{m} ; Z\right) \stackrel{h^{*}}{\longrightarrow} H^{2 m-4}\left(\wedge^{2} P_{m-2}^{m}, \Delta P_{m-2}^{m} ; Z\right)
\end{gathered}
$$

$h^{*}\left(\delta\left(\wedge z^{m-3} \wedge z^{m-2}\right)\right)=0$ in the lower line of diagram (4.2), while the upper line is injective, and the image of $S q^{2}$ in the upper right group is 0 . Thus 
$\Phi_{2}\left(\delta\left(\wedge z^{m-3} \wedge z^{m-2}\right)\right)=0$. This concludes the calculation of $C^{2} \pi_{(2 m-2) h}^{2 m-3}\left(\left(P^{m}\right)^{*}\right)$.

Proof of 4.8. We prove (2) only; the proofs of (1) and (3) are similar. By Lefschetz duality and Proposition 4.5

$$
\begin{aligned}
& H^{2 m-3}\left(\wedge^{2} P^{m}, \Delta P^{m} ; Z_{2}\right) \cong H^{3}\left(\left(P^{m}\right)^{*} ; Z_{2}\right) \cong Z_{2} \oplus Z_{2} \oplus Z_{2} \oplus Z_{2} \\
& H^{2 m-2}\left(\wedge^{2} P^{m}, \Delta P^{m} ; Z_{2}\right) \cong H^{2}\left(\left(P^{m}\right)^{*} ; Z_{2}\right) \cong Z_{2} \oplus Z_{2} \oplus Z_{2} .
\end{aligned}
$$

Using Theorem 11 of [11], it is easily seen that $\wedge z^{m-3} \wedge z^{m}, \wedge z^{m-2} \wedge z^{m-1}$, $u^{m-3} \wedge z^{m}, u^{m-2} \wedge z^{m-1}$ must be a $Z_{2}$-basis of $H^{2 m-3}\left(\wedge^{2} P^{m}, \Delta P^{m} ; Z_{2}\right)$ and $\wedge z^{m} \wedge z^{m-2}, \quad \wedge z^{m-1} \wedge z^{m-1}, \quad u^{m-2} \wedge z^{m}$ must be a $Z_{2}$-basis of $H^{2 m-2}\left(\wedge^{2} P^{m}, \Delta P^{m} ; Z_{2}\right)$.

In the next section (see Diagram 5.1) we show that $H^{*}\left(\wedge^{2} P^{m}, \Delta P^{m}\right) \stackrel{j^{*}}{\rightarrow} H^{*}\left(\left(P^{m}\right)^{*}\right) \stackrel{J^{*}}{\rightarrow} H^{*}\left(P\left(P^{m}\right)\right)$ is exact. Since $u y_{1}^{2^{r+1}-4} y_{2}^{s}+$ $y_{1}^{2^{r+1}-3} y_{2}^{s}=S q^{1}\left(u y_{1}^{2^{r+1}-5} y_{2}^{s}+y_{1}^{2^{r+1}-4} y_{2}^{s}\right)$ and $J^{*}\left(u y_{1}^{2^{r+1}-5}+y_{1}^{2^{r+i_{-4}}} y_{2}^{s}\right)=0$, there is an element $c$ in $\operatorname{ker}\left(S q^{1}\right) \subset H^{2 m-3}\left(\wedge^{2} P^{m}, \Delta P^{m} ; Z_{2}\right)$ which is mapped by $j^{*}$ to $\quad u y_{1}^{2^{r+1}-4} y_{2}^{s}+y_{1}^{2^{r+1}-3} y_{2}^{s}$. But $S q^{1}\left(u^{m-2} \wedge z^{m-1}\right)=S q^{1}\left(\wedge z^{m-3} \wedge z^{m}\right)=0$, $S q^{1}\left(\wedge z^{m-2} \wedge z^{m-1}\right)=\wedge z^{m-1} \wedge z^{m-1}, S q^{1}\left(u^{m-3} \wedge z^{m}\right)=u^{m-2} \wedge z^{m}$. So $c$ must be a linear combination of $u^{m-2} \wedge z^{m-1}$ and $z^{m-3} \wedge z^{m}$. Since, by Lemma 6 of [11], $j^{*}\left(u^{m-2} \wedge z^{m-1}\right)=0, j^{*}$ must map $\wedge z^{m-3} \wedge z^{m}$ to $u y_{1}^{2^{r+1}-4} y_{2}^{s}+y_{1}^{2^{r+1}-3} y_{2}^{s}$.

5. Calculation of $\boldsymbol{J}^{*}$. Let $(X, A)$ be a finite dimensional C.W. pair with inclusion $j: A \rightarrow X$, and let $\xi$ be a vector bundle over $X$. Assume $i \geqq \operatorname{dim} X-2$. In order to compute $J^{*}$, we first consider the more general problem of calculating $b=j^{*}: \pi_{\xi}^{i}(X) \rightarrow \pi_{\xi}^{i}(A)$.

Let $b^{s}: F^{s} \pi_{\xi}^{i}(X) / F^{s+1} \pi_{\xi}^{i}(X) \rightarrow F^{s} \pi_{\xi}^{i}(A) / F^{s+1} \pi_{\xi}^{i}(A)$ for $s=0,1,2$ be induced by $b$. To calculate $b$, in addition to the $b^{s}$, we need the following maps which are also induced by $b$

$$
\begin{aligned}
& \bar{b}^{0}: \operatorname{ker} b^{0} \rightarrow \text { coker } b^{1}=F^{1} \pi_{\xi}^{i}(A) /\left(F^{s} \pi_{\xi}^{i}(A)+b\left(F^{1} \pi_{\xi}^{i}(X)\right)\right) \\
& \bar{b}^{1}: \operatorname{ker} b^{1} \rightarrow \text { coker } b^{2}=F^{2} \pi_{\xi}^{i}(A) / b\left(F^{2} \pi_{\xi}^{i}(X)\right) \\
& \overline{\bar{b}}^{0}: \operatorname{ker} \bar{b}^{0} \rightarrow \text { coker } \bar{b}^{1}=F^{2} \pi_{\xi}^{i}(A) /\left(F^{2} \pi_{\xi}^{i}(A) \cap b\left(F^{1} \pi_{\xi}^{i}(X)\right)\right) .
\end{aligned}
$$

These homomorphisms together with Propositions 4.1 and 4.3 determine $\operatorname{ker} b$ (up to isomorphism) since

$\operatorname{ker} b \cap F^{2} \pi_{\xi}^{i}(X)=\operatorname{ker} b^{2}$;

$\left(\operatorname{ker} b \cap F^{1} \pi_{\xi}^{i}(X)\right) /\left(\operatorname{ker} b \cap F^{2} \pi_{\xi}^{i}(X)\right)=\operatorname{ker} \bar{b}^{1}$;

$\left(\operatorname{ker} b \cap F^{0} \pi_{\xi}^{i}(X)\right) /\left(\operatorname{ker} b \cap F^{1} \pi_{\xi}^{i}(X)\right)=\operatorname{ker} \overline{\bar{b}}^{0}$. 
They also determine coker $b$ since coker $\overline{\bar{b}}^{0}$, coker $\bar{b}^{0}$, coker $b^{0}$ give the quotients of the filtration:

$$
\left(F^{2} \pi_{\xi}^{i}(X)+i m b\right) / i m b \subset\left(F^{1} \pi_{\xi}^{i}(X)+\operatorname{im} b\right) / i m b \subset F^{0} \pi_{\xi}^{i}(X) / i m b
$$

Let $\alpha_{2}(B O)$ and $\Phi_{2}(B O)$ be as defined in $\S 4$, and let $\left(\alpha_{2}(B O) \rho\right)_{j}$ and $\left(\alpha_{2}(B O)\right)_{j}$ be functional operations in the category of spaces over $B O$ as defined in [14, p. 197]. Then $\left(\alpha_{2}(B O) \rho\right) j$ twisted by $\xi$ is defined by $\left(\alpha_{2}(\xi) \rho\right)_{j} x=\tilde{\delta}^{-1}\left(\alpha_{2}(\xi) \rho\right) k^{*-1} x$ for $x \in H^{*}\left(X ; \Gamma_{\xi}\right)$ and $\left(\alpha_{2}(B O)\right)_{j}$ twisted by $\xi$ is defined by $\left(\alpha_{2}(\xi)\right)_{j} y=\tilde{\delta}^{-1}\left(\alpha_{2}(\xi)\right) k^{*-1} y$ for $y \in H^{*}\left(X ; Z_{2}\right)$, where $k: X \rightarrow(X, A)$ is the inclusion and $\tilde{\delta}: H^{*}(A) \rightarrow H^{*}(X, A)$ is the coboundary operator.

Define $\left(\Phi_{2}(B O)\right)_{j}$ twisted by $\xi$ by

$$
\left(\Phi_{2}(\xi)\right)_{j} x=\tilde{\delta}^{-1}\left(\Phi_{2}(\xi)\right)\left(\left(\operatorname{ker}\left(\alpha_{2}(\xi) \rho\right)\right) \cap k^{*-1} x\right) \quad \text { for } \quad x \in H^{*}\left(X ; \Gamma_{\xi}\right) .
$$

The following proposition is adequate to compute all of the horizontal maps in diagram (4.1).

Proposition 5.1. After the obvious identifications

(1) $\bar{b}^{0}=\left(\alpha_{2}(\xi) \rho\right)_{j}$;

(2) if $C^{1} \pi_{\xi}^{i}(A)=0, \bar{b}^{1}$ is the map induced by $\left(\alpha_{2}(\xi)\right)_{j}$;

(3) if $C^{1} \pi_{\xi}^{i}(A)=0, \quad \bar{b}^{0}=\left(\Phi_{2}(\xi)\right)_{j}$ modulo the indeterminacy of $\left(\Phi_{2}(\xi)\right)_{j}$.

5.1 can be proved by looking at the first three stages of the standard Postnikov factorization of a sphere bundle and using the alternate definition of functional operation as given, for example, in [17, Ch. 16] or [14, p. 197]. It is essentially a tautology. (See [18] for more details.)

Before we can caclulate the maps $J^{*}$ in Diagram (4.1), we need the following explicit description of $J$. Let $V_{m+1,2}$ be the Stiefel manifold of orthonormal 2-frames in $R^{m+1}$. If we identify $\left(u_{1}, u_{2}\right),\left(-u_{1}, u_{2}\right)$, $\left(u_{1},-u_{2}\right)$, and $\left(-u_{1},-u_{2}\right)$ for any $\left(u_{1}, u_{2}\right)$ in $V_{m+1,2}$ the resulting quotient space is $P\left(P^{m}\right)$. Let $\pi: V_{m+1,2} \rightarrow P\left(P^{m}\right)$ be the quotient map. Define $\bar{g}: P\left(P^{m}\right) \rightarrow P^{m} \times P^{m}-\Delta P^{m}$ by $\bar{g}\left(\pi\left(u_{1}, u_{2}\right)\right)=\left(\left[u_{1},\right],\left[u_{2}\right]\right)$, where $[u]$ is the element of $P^{m}$ determined by the nonzero vector $u$. It is shown in [8] that $\bar{g}$ is a homotopy equivalence.

Let $p: P^{m} \times P^{m}-\Delta P^{m} \rightarrow\left(P^{m}\right)^{*}$ be the projection and set $S Z_{m+1,2}=$ $p \bar{g}\left(P\left(P^{m}\right)\right) \subset\left(P^{m}\right)^{*}$. We have a commutative diagram

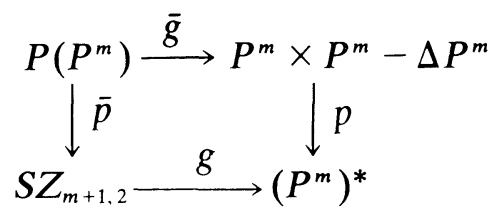


where $g$ is the inclusion and $\bar{p}=p \mid P\left(P^{m}\right)$. Then $\bar{p}$ is a double covering and $g, \bar{g}$ are homotopy equivalences. (See 2.5 of [8].)

Define a homeomorphism $f: S Z_{m+1,2} \rightarrow S Z_{m+1,2}$ by $f\left(p\left(\left[v_{1}\right],\left[v_{2}\right]\right)\right)=$ $p\left(\left[v_{1}+v_{2}\right],\left[v_{1}-v_{2}\right]\right)$, for any unit vectors $v_{1}$ and $v_{2}$.

\section{Proposition 5.2. J is homotopic to $g f \bar{p}: P\left(P^{m}\right) \rightarrow\left(P^{m}\right)^{*}$.}

Proof. It is clear that there is a metric $d$ on $S^{m}$ left invariant by the antipodal map, such that if $\exp _{d}$ is the exponential map defined with respect to $d$, we have $\exp _{d}\left(v_{1}, v_{2}\right)=(1 / \sqrt{2})\left(v_{1}+v_{2}\right)$ for $\left(v_{1}, v_{2}\right) \in V_{m+1,2}$ where we have identified $V_{m+1,2}$ with the tangent sphere bundle of $S^{m}$. Now $d$ induces a metric, $\bar{d}$, on $P^{m}$. Define $\bar{J}: P\left(P^{m}\right) \rightarrow\left(P^{m}\right)^{*}$ by $\bar{J}([v])=\left[\exp _{\bar{d}}(v), \exp _{\bar{d}}(-v)\right]$ for $v$ a unit tangent vector of $P^{m}$. Then $\bar{J}=g f \bar{p}$ and $\bar{J}$ is clearly homotopic to $J$ (as defined in $\S 2$ ).

PROPOSITION 5.3.

(1) $J^{*}(u)=J^{*}\left(y_{1}\right)=v$;

(2) $J^{*}\left(y_{2}\right)=z^{2}+z v$.

Proof. $J^{*}(u)=v$ since $J$ is covered by a map of double covers. Since by $4.5, u^{2}=u y_{1}$, we must also have $J^{*}\left(y_{1}\right)=v$. It is shown in [8] that $p^{*}\left(y_{2}\right) \neq 0$. By 5.2, this implies $J^{*}\left(y_{2}\right) \neq 0$. Since $S q^{1} y_{2}=y_{1} y_{2}$, $S q^{1}\left(J^{*}\left(y_{2}\right)\right)=v J^{*}\left(y_{2}\right)$. The only non-zero element of $H^{2}\left(P\left(P^{m}\right) ; Z_{2}\right)$ with this property is $z^{2}+z v$; hence, $J^{*}\left(y_{2}\right)=z^{2}+z v$.

Turning now to the calculation of $J^{*}$ in Diagram (4.1), we again indicate the details in the case $m \equiv 3 \bmod 4$ only.

$J^{*}: \pi_{2 m h}^{2 m-1}\left(\left(P^{m}\right)^{*}\right) \rightarrow \pi_{2 m h}^{2 m-1}\left(P\left(P^{m}\right)\right) \quad$ is the 0 -map because $J^{*}\left(u y_{1}^{2^{r+1}-2} y_{2}^{s}\right)=v^{2^{r+1}-1}\left(z^{2}+z v\right)^{s}=0$ by 5.3 and 4.4 .

The calculation of $J^{*}$ on $\pi_{(2 m-1) h}^{2 m-2}\left(\left(P^{m}\right)^{*}\right)$ is similar to (and easier than) the calculation of $J^{*}$ on $\pi_{(2 m-2) h}^{2 m-3}\left(\left(P^{m}\right)^{*}\right)$. So we include the computation of the latter only.

It follows directly from the computations of $\S 4$ and Proposition 5.3 that

$$
\begin{gathered}
F^{0} \pi_{(2 m-2) h}^{2 m-3}\left(\left(P^{m}\right)^{*}\right) / F^{1} \pi_{(2 m-2) h}^{2 m-3}\left(\left(P^{m}\right)^{*}\right) \cong Z_{2} \oplus Z_{2} \\
\qquad J^{* 0} \\
F^{0} \pi_{(2 m-2) h}^{2 m-3}\left(P\left(P^{m}\right)\right) / F^{1} \pi_{(2 m-2) h}^{2 m-3}\left(P\left(P^{m}\right)\right) \cong Z_{2} \oplus Z_{2}
\end{gathered}
$$

has image $Z_{2}$ generated by $z^{m-2} v^{m-1}+z^{m-1} v^{m-2}$ and kernel $Z_{2}$ generated by $\delta\left(u y_{1}^{2^{r+1-5}} y_{2}^{s}+y_{1}^{2^{r+1-4}} y_{2}^{s}\right)$, and

$$
\begin{gathered}
F^{1} \pi_{(2 m-2) h}^{2 m-3}\left(\left(P^{m}\right)^{*}\right) / F^{2} \pi_{(2 m-2) h}^{2 m-3}\left(\left(P^{m}\right)^{*}\right) \cong Z_{2} \oplus Z_{2} \\
\downarrow^{* 1} J^{* 1} \\
F^{1} \pi_{(2 m-2) h}^{2 m-3}\left(P\left(P^{m}\right)\right) / F^{2} \pi_{(2 m-2) h}^{2 m-3}\left(P\left(P^{m}\right)\right) \cong Z_{2} \oplus Z_{2}
\end{gathered}
$$


has image $Z_{2}$ generated by $z^{m-2} v^{m}+z^{m-1} v^{m-1}$ and kernel $Z_{2}$ generated by $u y_{1}^{2^{r+1}-3} y_{2}^{s}+y_{1}^{2^{r+1}-2} y_{2}^{s}$, while

$$
\begin{gathered}
F^{2} \pi_{(2 m-2) h}^{2 m-3}\left(\left(P^{m}\right)^{*}\right) \cong Z_{2} \\
\downarrow^{* 2} J^{* 2} \\
F^{2} \pi_{(2 m-2) h}^{2 m-3}\left(P\left(P^{m}\right)\right) \cong Z_{2}
\end{gathered}
$$

is the 0-map.

Recall that $\wedge^{2} P^{m}$ is the set of unordered pairs in $P^{m}$, and we have $\left(P^{m}\right)^{*} \subset \wedge^{2} P^{m}$ and $\Delta P^{m} \subset \wedge^{2} P^{m}$. The complement of $J\left(P\left(P^{m}\right)\right)$ in $\wedge^{2} P^{m}$ has two components; let $N$ be the closure of that containing $\Delta P^{m}$. Then $N$ is a tubular neighborhood of $\Delta P^{m}$. There is a commutative diagram

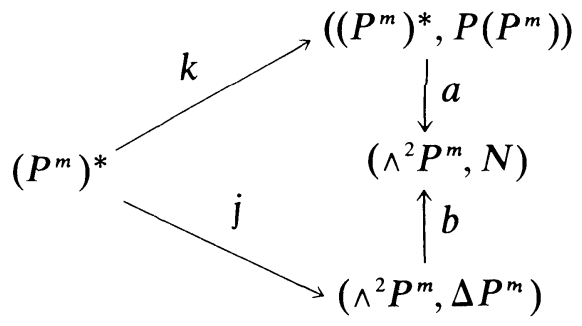

where all the maps are inclusions. Since both $a$ and $b$ induce isomorphisms of cohomology groups, we may replace $k$ by $j$ in the computation of the functional operations of Proposition 5.1. The advantage in this is that $H^{*}\left(\wedge^{2} P^{m}, \Delta P^{m} ; Z_{2}\right)$ is completely described in [11]. (See Lemma 10 of [11] for the action of the Steenrod algebra on $H^{*}\left(\wedge^{2} P^{m}, \Delta P^{m} ; Z_{2}\right)$.)

We return now to the computation of $J^{*}$ on $\pi_{(2 m-2) h}^{2 m-3}\left(\left(P^{m}\right)^{*}\right)$.

Proposition 5.4.

$$
\begin{aligned}
& \left(\alpha_{2}((2 M-2) h)\right)_{J}\left(u y_{1}^{2^{r+1}-3} y_{2}^{s}+y_{1}^{2^{r+1}-2} y_{2}^{s}\right)=0 ; \\
& \left(\alpha_{2}((2 m-2) h) \rho\right)_{J}\left(\delta\left(u y_{1}^{2^{r+1}-5} y_{2}^{s}+y_{1}^{2^{r+1}-4} y_{2}^{s}\right)\right)=0 \\
& \left(\Phi_{2}((2 m-2) h)\right)_{J}\left(\delta\left(u y_{1}^{2^{r+1}-5} y_{2}^{s}+y_{1}^{2^{r+1-4}} y_{2}^{s}\right)\right)=0
\end{aligned}
$$

with 0 indeterminacy.

Proof. Recall (Lemma 4.8) that

$$
\begin{aligned}
& j^{*}\left(\wedge z^{m-3} \wedge z^{m}\right)=u y_{1}^{2^{r+1}-4} y_{2}^{s}+y_{1}^{2^{r+1}-3} y_{2}^{s}: \\
& j^{*}\left(\wedge z^{m-2} \wedge z^{m}\right)=u y_{1}^{2^{r+1}-3} y_{2}^{s}+y_{1}^{2^{r+1}-2} y_{2}^{s} .
\end{aligned}
$$

Since $S q^{1}\left(\wedge z^{m-4} \wedge z^{m}\right)=\wedge z^{m-3} \wedge z^{m}$, we have 


$$
j^{*}\left(\delta\left(\wedge z^{m-4} \wedge z^{m}\right)\right)=\delta\left(u y_{1}^{2 r+1-5} y_{2}^{s}+y_{1}^{2^{r+1}-4} y_{2}^{s}\right) .
$$

Also, $\alpha_{2}((2 m-2) h)=S q^{2}$ and $\Phi_{2}((2 m-2) h)=\Phi_{2}$ (untwisted). Then $\left(\alpha_{2}((2 m-2) h) \rho\right)_{J}\left(\delta\left(u y_{1}^{r^{r+1}-5} y_{2}^{s}+y_{1}^{2^{r+1}-4} y_{2}^{s}\right)\right)=0$ since $S q^{2}\left(\rho \delta\left(\wedge z^{m-4} \wedge z^{m}\right)\right)$ $=S q^{2}\left(\wedge z^{m-3} \wedge z^{m}\right)=0$, while $\left(\alpha_{2}((2 m-2) h)\right)_{J}\left(u y_{1}^{2^{r+1}-3} y_{2}^{s}+y_{1}^{2^{2+1}-2} y_{2}^{s}\right)=0$ because $S q^{2}\left(\wedge z^{m-2} \wedge z^{m}\right)=0$. The last assertion of Proposition 5.4 follows immediately from:

PROPOSITION 5.5. $\Phi_{2}\left(\delta\left(\wedge z^{m-4} \wedge z^{m}\right)\right)=0 \in H^{2 m}\left(\wedge^{2} P^{m}, \Delta P^{m} ; Z_{2}\right)$ with indeterminacy 0 .

Proof. Recall from $\S 4$ the map $g:\left(\wedge^{2} P^{m}, \Delta P^{m}\right) \rightarrow\left(\wedge^{2} P_{m-3}^{m}, \Delta P_{m-3}^{m}\right)$. By part (vi) of theorem 20 in [11], $\wedge z^{m-3} \wedge z^{m}$ in $H^{2 m-3}\left(\wedge^{2} P_{m-3}^{m}, \Delta P_{m-3}^{m} ; Z_{2}\right)$ is the reduction of some integer class $x$ (because $z^{m-3}$ and $z^{m}$ are both reductions of integer classes in $\left.H^{*}\left(P_{m-3}^{m}\right)\right)$. Then $g^{*} x=\delta\left(\wedge z^{m-4} \wedge z^{m}\right)$ (because $\rho \delta\left(\wedge z^{m-4} \wedge z^{m}\right)=\wedge z^{m-3} \wedge z^{m}$ in $H^{2 m-3}\left(\wedge^{2} P^{m}, \Delta P^{m} ; Z_{2}\right)$ ).

Now $P_{m-3}^{m}$ has the same homotopy type as $P_{m-3}^{m-1} \vee S^{m}$ (see Ch. 15 of [9]). Let $r: S^{m} \rightarrow P_{m-3}^{m}$ be the inclusion of $S^{m}$ in $P_{m-3}^{m-1} \vee S^{m}$ followed by a homotopy equivalence. We have a commutative diagram

$$
\begin{gathered}
H^{2 m}\left(\wedge^{2} S^{m}, \Delta S^{m} ; Z_{2}\right) \stackrel{r^{*}}{\longleftarrow} H^{2 m}\left(\wedge^{2} P_{m-3}^{m}, \Delta P_{m-3}^{m} ; Z_{2}\right) \\
\bigcap_{2} \Phi_{2} \\
H^{2 m-3}\left(\wedge^{2} S^{m}, \Delta S^{m} ; Z\right) \stackrel{r^{*}}{\longleftarrow} H^{2 m-3}\left(\wedge^{2} P_{m-3}^{m}, \Delta P_{m-3}^{m} ; Z\right) .
\end{gathered}
$$

Now $r^{*} x=0$ in the lower line of (5.2) because $r^{*}\left(\wedge z^{m-3} \wedge z^{m}\right)=0$ in $H^{2 m-3}\left(\wedge^{2} S^{m}, \Delta S^{m} ; Z_{2}\right)$ and

$$
\rho: H^{2 m-3}\left(\wedge^{2} S^{m}, \Delta S^{m} ; Z\right) \rightarrow H^{2 m-3}\left(\wedge^{2} S^{m}, \Delta S^{m} ; Z_{2}\right)
$$

is injective. Furthermore, $r^{*}$ is an isomorphism in the top line, and the image of $S q^{2}$ in $H^{2 m}\left(\wedge^{2} S^{m}, \Delta S^{m} ; Z_{2}\right)$ is 0 . Hence $\Phi_{2}(x)=0$ and $\Phi_{2}\left(\delta\left(\wedge z^{m-4} \wedge z^{m}\right)\right)=0$ in $H^{2 m}\left(\wedge^{2} P^{m}, \Delta P^{m} ; Z_{2}\right)$ since $g^{*} x=\delta\left(\wedge z^{m-4} \wedge z^{m}\right)$. It is easily checked that the indeterminacy is 0 . This completes the proof of 5.5.

By Propositions 5.1 and 5.4, $J^{*}: \pi_{(2 m-2) h}^{2 m-3}\left(\left(P^{m}\right)^{*}\right) \rightarrow \pi_{(2 m-2) h}^{2 m-3}\left(P\left(P^{m}\right)\right)$ has kernel and cokernel each of order 8 . Utilizing Proposition 4.3 and computing

$$
\begin{aligned}
& \alpha_{2}((2 m-2) h) \rho \delta\left(u y_{1}^{2^{r+1}-5} y_{2}^{s}+y_{1}^{2^{r+1}-4} y_{2}^{s}\right)=u y_{1}^{2^{r+1}-3} y_{2}^{s}+y_{1}^{2^{r+1}-2} y_{2}^{s} \\
& \alpha_{1}((2 m-2) h)\left(u y_{1}^{2^{r+1}-3} y_{2}^{s}+y_{1}^{2^{r+1}-2} y_{2}^{s}\right)=u y_{1}^{2^{r+1}-2} y_{2}^{s}
\end{aligned}
$$


we see that ker $J^{*}$ is actually isomorphic to $Z_{8}$. In a similar manner, we find that coker $J^{*} \cong Z_{8}$. This determines $J^{*}$ up to isomorphism.

6. Calculation of $\cup \gamma^{1}\left(P^{m}\right)$ and $\cup \zeta^{1}\left(P^{m}\right)$. Again, let $(X, A)$ be a finite dimensional C.W. pair and let $\xi$ be a vector bundle over $X$. Let $\eta$ be a line bundle over $X$; and recall ( 81 ) the cup product $\pi_{\xi}^{i}(X, A) \otimes \pi_{\eta}^{i}(X) \rightarrow \pi_{\xi \oplus \eta}^{i+j}(X, A)$. Thus $\cup \gamma$ is a homomorphism $\pi_{\xi}^{i}(X, A) \rightarrow \pi_{\xi \oplus \eta}^{i+1}(X, A)$, where $\gamma$ is the single obstruction to a section of $\eta$.

Proposition 6.1. Let

$$
\theta_{s}: H^{i+s}\left(X, A ; \pi_{s} \otimes \Gamma_{\xi}\right) \rightarrow H^{i+s+1}\left(X, A ; \pi_{s} \otimes \Gamma_{\xi \oplus \eta}\right)
$$

be cup product with the (ordinary) Euler class of $\eta$. Then

$$
\begin{aligned}
& \left(F^{s} \pi_{\xi}^{i}(X, A)\right) \cup \gamma \subset F^{s} \pi_{\xi \oplus \eta}^{i+1}(X, A) \\
& \theta_{s}\left(C^{s} \pi_{\xi}^{i}(X, A)\right) \subset C^{s} \pi_{\xi \oplus \eta}^{i+1}(X, A) \\
& \theta_{s}\left(D^{s} \pi_{\xi}^{i}(X, A)\right) \subset D^{s} \pi_{\xi \oplus \eta}^{i+1}(X, A)
\end{aligned}
$$

and the following diagram is commutative

$$
\begin{gathered}
F^{s} \pi_{\xi}^{i}(X, A) / F^{s+1} \pi_{\xi}^{i}(X, A) \stackrel{d_{i}^{s+i}}{\longrightarrow} D^{s} \pi_{\xi}^{i}(X, A) / C^{s} \pi_{\xi}^{i}(X, A) \\
\sum^{s} \pi_{\xi \gamma}^{i+1}(X, A) / F^{s+1} \pi_{\xi \oplus \eta}^{i+1}(X, A) \stackrel{d_{i+1}^{s+i+1}}{\longrightarrow} D^{s} \pi_{\xi \oplus \eta}^{i+1}(X, A) / C^{s} \pi_{\xi \oplus \eta}^{i+1}(X, A)
\end{gathered}
$$

where $\overline{\cup \gamma}$ and $\bar{\theta}_{s}$ are induced by $\cup \gamma$ and $\theta_{s}$ respectively.

Proof. Let $Y$ be the total space of the sphere bundle of $\eta$. Then $\cup \gamma$ is the composite $\pi_{\xi}^{i}(X, A) \stackrel{\cup \dot{U}_{h}}{\longrightarrow} \pi_{\xi \oplus \eta}^{i+1}(X, A \cup Y) \rightarrow \pi_{\xi \oplus \eta}^{i+1}(X, A)$ where the first map is the Thom isomorphism of $\S 1$ and the second map is induced by the inclusion $(X, A) \subset(X, A \cup Y)$. Since the AtiyahHirzebruch spectral sequence is functorial, it suffices to show that $\cup U_{h}$ "preserves" the Atiyah-Hirzebruch spectral sequence in the obvious way. $\left(C^{s} \pi_{\xi}^{i}(X, A)\right.$ and $D^{s} \pi_{\xi}^{i}(X, A)$ are mapped to $C^{s} \pi_{\xi \oplus \eta}^{i+1}(X, A \cup Y)$ and $D^{s} \pi_{\xi \oplus \eta}^{i+1}(X, A \cup Y)$, respectively, by the ordinary Thom isomorphism.) But this is not difficult, if one uses the definition of the Atiyah-Hirzebruch spectral sequence given in [3, §4]. We omit the proof. (A detailed exposition can be found in [18].)

It is now a trivial matter to compute the maps 


$$
\begin{aligned}
& F^{s} \pi_{n h}^{n-1}\left(\left(P^{m}\right)^{*}\right) / F^{s+1} \pi_{n h}^{n-1}\left(\left(P^{m}\right)^{*}\right) \rightarrow F^{s} \pi_{(n+1) h}^{n}\left(\left(P^{m}\right)^{*}\right) / F^{s+1} \pi_{(n+1) h}^{n}\left(\left(P^{m}\right)^{*}\right) \\
& F^{s} \pi_{n h}^{n-1}\left(P\left(P^{m}\right)\right) / F^{s+1} \pi_{n h}^{n-1}\left(P\left(P^{m}\right)\right) \rightarrow F^{s} \pi_{(n+1) h}^{n}\left(P\left(P^{m}\right)\right) / F^{s+1} \pi_{(n+1) h}^{n}\left(P\left(P^{m}\right)\right)
\end{aligned}
$$

These maps need not determine the maps $\cup \gamma^{1}\left(P^{m}\right)$ and $\cup \zeta^{1}\left(P^{m}\right)$ up to isomorphism (secondary homomorphisms of the sort discussed in $\S 5$ could play a role); however, in all of the cases of concern to us in this paper, the above maps do determine those cup products.

As an example, we compute $\cup \gamma^{1}\left(P^{m}\right)$ on $\pi_{(2 m-2) h}^{2 m-3}\left(\left(P^{m}\right)^{*}\right)$. Now $Z_{2} \oplus Z_{2} \cong D^{0} \pi_{(2 m-2) h}^{2 m-3}\left(\left(P^{m}\right)^{*}\right) \rightarrow D^{0} \pi_{(2 m-1) h}^{2 m-2}\left(\left(P^{m}\right)^{*}\right) \cong Z_{2}$ is onto, with kernel generated by $\delta\left(u y_{1}^{2^{r+1}-5} y_{2}^{s}+y_{1}^{2^{r+1}-4} y_{2}^{s}\right)$, while

$$
D^{1} \pi_{(2 m-2) h}^{2 m-3}\left(\left(P^{m}\right)^{*}\right) / C^{1} \pi_{(2 m-2) h}^{2 m-3}\left(\left(P^{m}\right)^{*}\right) \rightarrow D^{1} \pi_{(2 m-1) h}^{2 m-2}\left(\left(P^{m}\right)^{*}\right) / C^{1} \pi_{(2 m-1) h}^{2 m-2}\left(\left(P^{m}\right)^{*}\right)
$$

is onto with kernel generated by $u y_{1}^{2^{r+1}-3} y_{2}^{s}+y_{1}^{2^{r+1}-2} y_{2}^{s}$. Now

$$
D^{2} \pi_{(2 m-2) h}^{2 m-3}\left(\left(P^{m}\right)^{*}\right) / C^{2} \pi_{(2 m-2) h}^{2 m-3}\left(\left(P^{m}\right)^{*}\right) \rightarrow D^{2} \pi_{(2 m-1) h}^{2 m-2}\left(\left(P^{m}\right)^{*}\right) / C^{2} \pi_{(2 m-1) h}^{2 m-2}\left(\left(P^{m}\right)^{*}\right)
$$

is trivially the 0 -map with kernel generated by $u y_{1}^{2^{r+1}-2} y_{2}^{s}$.

This establishes that $\cup \gamma^{1}\left(P^{m}\right)$ is onto $\pi_{(2 m-1) h}^{2 m-2}\left(\left(P^{m}\right)^{*}\right)$. The kernel of that map must be isomorphic to either $Z_{8}$ or $Z_{2} \oplus Z_{4}$. That it is actually isomorphic to $Z_{8}$ is shown by utilizing Proposition 4.3 and computing

$$
\begin{gathered}
\alpha_{2}((2 m-2) h) \rho \delta\left(u y_{1}^{2^{r+1}-5} y_{2}^{s}+y_{1}^{2^{r+1}-4} y_{2}^{s}\right)=u y_{1}^{2^{r+1}-3} y_{2}^{s}+y_{1}^{2^{r+1}-2} y_{2}^{s} \\
\alpha_{1}((2 m-2) h)\left(u y_{1}^{2^{r+1}-3} y_{2}^{s}+y_{1}^{r^{r+1}-2} y_{2}^{s}\right)=u y_{1}^{2^{r+1}-2} y_{2}^{s} .
\end{gathered}
$$

7. Closing remarks. The calculations of $\S 4, \S 5, \S 6$ are sufficient to determine the maps of diagram (0.1) up to isomorphism. It is then a simple matter to write down the complete diagram (except for the uncertainty in $\phi_{2 m-2}$ when $m \equiv 3 \bmod 4$ ). The reader should be cautioned that it need not be a simple matter, it just happens to be easy for projective spaces. For example, determining the relationship between $\operatorname{ker}\left(\phi_{2 m-2}\right)$ and $\operatorname{ker}\left(\mathscr{E}_{2 m-2}\right)$ in general requires further calculation. But when $m \neq 3 \bmod 4$, the triviality of the groups and the commutativity of diagram (0.1) makes such calculation unnecessary.

\section{REFERENCES}

1. D. Bausum, Embeddings and Immersions of Manifolds in Euclidean Space, Thesis, Yale University, 1974.

2. - Embeddings and immersion of manifolds in Euclidean space, Trans. Amer. Math. Soc., 213 (1975), 263-303.

3. J. C. Becker, Cohomology and the classification of liftings, Trans. Amer. Math. Soc., 133 (1968), $447-475$.

4. Extensions of cohomology theories, Illinois J. Math., 14 (1970), 551-584. 
5. A. Dold, Partitions of unity in the theory of fibrations, Ann. Math., 78 (1963), 223-255.

6. A. Haefliger, Plongements differentiable dans le domaine stable, Comm. Math. Helv., 37 (1962), 155-176.

7. A. Haefliger and M. Hirsch, Immersions in the stable range, Ann. Math., 75 (1962), 231-241.

8. D. Handel, An embedding theorem for real projective spaces, Topology, 7 (1968), 125-130.

9. D. Husemoller, Fiber Bundles, McGraw-Hill, 1966.

10. L. L. Larmore, Twisted cohomology theories and the single obstruction to lifting, Pacific J. Math., 41 (1972), 755-769.

11. The Cohomology of $\left(\wedge^{2} X, \Delta X\right)$, Canad. J. Math., 25 (1973), 908-921.

12. L. L. Larmore and P. E. Thomas, Group extensions and twisted cohomology theories, Illinois J. Math., 17 (1973), 397-410.

13. M. Mahowald, On extending cross-sections in orientable $V_{k+m, m}$ bundles, Bull. Amer. Math. Soc., 68 (1962), 596-602.

14. J. F. McClendon, Higher order twisted cohomology operations, Invent. Math., 7 (1969), $183-214$.

15. — Obstruction theory in fiber spaces, Math. Z., 120 (1971), 1-17.

16. — On stable fiber space obstructions, Pacific J. Math., 36 (1971), 439-445.

17. R. Mosher and M. Tangora, Cohomology Operations and Applications in Homotopy Theory, Harper and Row, 1968.

18. R. Rigdon, Immersions and Embeddings of Manifolds in Euclidean Space, dissertation, University of California at Berkeley, 1970.

19. C. A. Robinson, Stable homotopy theory over a fixed base space, Bull. Amer. Math. Soc., 80 (1974), 248-252.

Received March 24, 1975.

California State College-Dominguez Hills

AND

IUPUI 


\section{PACIFIC JOURNAL OF MATHEMATICS}

\section{EDITORS}

RICHARD ARENS (Managing Editor)

University of California

Los Angeles, California 90024

\author{
R. A. Beaumont \\ University of Washington \\ Seattle, Washington 98105
}

\section{J. DugunduI}

Department of Mathematics University of Southern California Los Angeles, California 90007

D. Gilbarg and J. Milgram

Stanford University

Stanford, California 94305

\section{ASSOCIATE EDITORS}

E. F. BECKENBACH
B. H. NeumanN

F. Wolp
K. YoshidA

\section{SUPPORTING INSTITUTIONS}

UNIVERSITY OF BRITISH COLUMBIA

CALIFORNIA INSTITUTE OF TECHNOLOGY

UNIVERSITY OF CALIFORNIA

MONTANA STATE UNIVERSITY

UNIVERSITY OF NEVADA

NEW MEXICO STATE UNIVERSITY

OREGON STATE UNIVERSITY

UNIVERSITY OF OREGON

OSAKA UNIVERSITY

\author{
UNIVERSITY OF SOUTHERN CALIFORNIA \\ STANFORD UNIVERSITY \\ UNIVERSITY OF HAWAII \\ UNIVERSITY OF TOKYO \\ UNIVERSITY OF UTAH \\ WASHINGTON STATE UNIVERSITY \\ UNIVERSITY OF WASHINGTON \\ AMERICAN MATHEMATICAL SOCIETY
}

The Supporting Institutions listed above contribute to the cost of publication of this Journal, but they are not owners or publishers and have no responsibility for its contents or policies.

Mathematical papers intended for publication in the Pacific Journal of Mathematics should be in typed form or offset-reproduced (not dittoed). double spaced with large margins. Underline Greek letters in red, German in green, and script in blue. The first paragraph or two must be capable of being used separately as a synopsis of the entire paper. Items of the biblography should not be cited there unless absolutely necessary, in which case they must be identified by author and Journal, rather than by item number. Manuscripts, in duplicate, may be sent to any one of the four editors. Please classify according to the scheme of Math. Reviews, Index to Vol. 39. All other communications should be addressed to the managing editor, or Elaine Barth, University of California, Los Angeles, California, 90024.

100 reprints are provided free for each article, only if page charges have been substantially paid. Additional copies may be obtained at cost in multiples of 50 .

The Pacific Journal of Mathematics is issued monthly as of January 1966. Regular subscription rate: $\$ 72.00$ a year (6 Vols., 12 issues). Special rate: $\$ 36.00$ a year to individual members of supporting institutions.

Subscriptions, orders for back numbers, and changes of address should be sent to Pacific Journal of Mathematics, 103 Highland Boulevard, Berkeley, California, 94708.

PUBLISHED BY PACIFIC JOURNAL OF MATHEMATICS, A NON-PROFIT CORPORATION Printed at Jerusalem Academic Press, POB 2390, Jerusalem, Israel.

Copyright (C) 1976 Pacific Journal of Mathematics All Rights Reserved 


\section{Pacific Journal of Mathematics}

\section{Vol. 64, No. $2 \quad$ June, 1976}

Richard Fairbanks Arnold and A. P. Morse, Plus and times............. 297

Edwin Ogilvie Buchman and F. A. Valentine, External visibility ......... 333

R. A. Czerwinski, Bonded quadratic division algebras.............. 341

William Richard Emerson, Averaging strongly subadditive set functions in unimodular amenable groups. II .........................

Lynn Harry Erbe, Existence of oscillatory solutions and asymptotic behavior for a class of third order linear differential equations ............. 369

Kenneth R. Goodearl, Power-cancellation of groups and modules........ 387

J. C. Hankins and Roy Martin Rakestraw, The extremal structure of locally compact convex sets ...................................

Burrell Washington Helton, The solution of a Stieltjes-Volterra integral

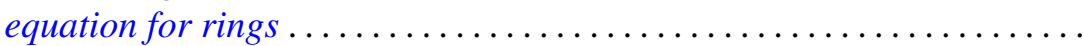

Frank Kwang-Ming Hwang and Shen Lin, Construction of 2-balanced



Wei-Eihn Kuan, Some results on normality of a graded ring ... 455

Dieter Landers and Lothar Rogge, Relations between convergence of series and convergence of sequences ......................... 465

Lawrence Louis Larmore and Robert David Rigdon, Enumerating immersions and embeddings of projective spaces ................

Douglas C. McMahon, On the role of an abelian phase group in relativized problems in topological dynamics..................

Robert Wilmer Miller, Finitely generated projective modules and TTF classes...

Yashaswini Deval Mittal, A class of isotropic covariance functions ...

Anthony G. Mucci, Another martingale convergence theorem ...

Joan Kathryn Plastiras, Quasitriangular operator algebras ...

John Robert Quine, Jr., The geometry of $p\left(S^{1}\right) \ldots \ldots \ldots$. 